\title{
Alternating Copolymerization of Carbon Dioxide with Epoxides Using Highly Active Dinuclear Nickel Complexes: Catalysis and
}

\author{
Kinetics \\ Yu-Chia Su, and Bao-Tsan Ko* \\ Department of Chemistry, National Chung Hsing University, Taichung 402, Taiwan \\ *To whom correspondence should be addressed.E-mail: btko@dragon.nchu.edu.tw (B.-T. \\ Ko), Tel: 886-4-22840411-715, Fax: 886-4-22862547.
}

Figure S1. ORTEP drawing of complex 1 with probability ellipsoids drawn at 30\% level. Figure S2. ORTEP drawing of complex 2 with probability ellipsoids drawn at $40 \%$ level. Figure S3. ORTEP drawing of complex 3 with probability ellipsoids drawn at 30\% level. Figure S4. ORTEP drawing of complex 4 with probability ellipsoids drawn at 50\% level. Figure S5. ORTEP drawing of complex 5 with probability ellipsoids drawn at 50\% level. Figure S6. ORTEP drawing of complex 6 with probability ellipsoids drawn at 30\% level. Figure S7. ORTEP drawing of complex 8 with probability ellipsoids drawn at $30 \%$ level. Figure S8. ORTEP drawing of complex 9 with probability ellipsoids drawn at 30\% level. Figure S9. ORTEP drawing of complex 10 with probability ellipsoids drawn at 30\% level. Figure S10. ORTEP drawing of complex 11 with probability ellipsoids drawn at 50\% level. Figure S11. GPC traces for the afforded PCHC with a bimodal molecular weight distribution catalyzed by dinickel complex 6 (Table 2, entry 4).

Figure S12. The plot of $M_{\mathrm{n}}(\square)$ and $D(\square)$ (determined from GPC analysis) versus CHO conversion for the $\mathrm{CO}_{2}$-copolymerization of $\mathrm{CHO}$ using dinickel complex $\mathbf{6}$ as the catalyst $\left([\mathrm{CHO}]_{0} /[6]_{0}=5000\right)$ at $80{ }^{\circ} \mathrm{C}$ and 20.7 bar $\mathrm{CO}_{2}$. 
Figure S13. Carbonyl region of ${ }^{13} \mathrm{C}$ NMR spectra of the PCHC copolymer (Table 2, entry 4) in $\mathrm{CDCl}_{3}$. (inset) Expanded carbonyl region.

Figure S14. ${ }^{1} \mathrm{H}$ NMR spectrum of the purified copolymer produced by utilizing dinickel complex 6 (Table 3, entry 2) in $\mathrm{CDCl}_{3}$. The peak at $\delta 4.65 \mathrm{ppm}$ is assigned to the methine protons in PCHC, and no significant signal at 3.3-3.5 ppm suggests $>99 \%$ carbonate carbonates in PCHC.

Figure S15. The plot of $M_{\mathrm{n}}(\square)$ and $Ð(\square)$ (determined from GPC analysis) versus $\mathrm{CHO}$ conversion for the $\mathrm{CO}_{2}$-copolymerization of $\mathrm{CHO}$ employing dinickel complex 6 as the catalyst $\left([\mathrm{CHO}]_{0} /[6]_{0}=1600\right)$ at $100{ }^{\circ} \mathrm{C}$ and 1 atm of $\mathrm{CO}_{2}$ pressure.

Figure S16. The plot of $M_{\mathrm{n}}(\boldsymbol{\square})$ and PDI ( $\left.\boldsymbol{\Delta}\right)$ (determined from GPC analysis) versus VCHO conversion for the $\mathrm{CO}_{2}$-copolymerization of $\mathrm{VCHO}$ using dinickel complex $\mathbf{6}$ as the catalyst $\left([\mathrm{CHO}]_{0} /[6]_{0}=5000\right)$ at $80{ }^{\circ} \mathrm{C}$ and 20.7 bar $\mathrm{CO}_{2}$.

Figure S17. GPC traces for the produced PCHC with a unimodal molecular weight distribution catalyzed by dinickel complex 6 (Table 4, entry 8).

Figure S18. The MALDI-TOF spectrum of the obtained PCHC catalyzed by dinickel complex 6 with the addition of water as the chain transfer agent (Table 4, entry 8).

Figure S19. The MALDI-TOF spectrum of the afforded PCHC polyol catalyzed by dinickel complex 6 (Table 4, entry 9).

Figure S20. The MALDI-TOF spectrum of the yielded PVCHC polyol catalyzed by dinickel complex 6 (Table 4, entry 10).

Figure S21. The MALDI-TOF spectrum of the produced PCPC polyol catalyzed by dinickel complex 6 (Table 4, entry 11).

Figure S22. Plots of TOFs with different initial $\mathrm{CO}_{2}$ pressures utilizing dinickel complex 6 $(0.02 \mathrm{~mol} \%)$ at $80{ }^{\circ} \mathrm{C}$ for $4 \mathrm{~h}$.

Figure S23. The kinetic plot of $\ln \left([\mathrm{CHO}]_{0} /[\mathrm{CHO}]_{t}\right)$ versus time for the copolymerization of $\mathrm{CHO}$ and $\mathrm{CO}_{2}$ by dinickel complex 6 at $80{ }^{\circ} \mathrm{C}$ and an initial $\mathrm{CO}_{2}$ pressure of 20.7 bar using 
a catalyst loading of $0.02 \mathrm{~mol} \%$.

Figure S24. A linear plot of $\ln k_{\mathrm{obs}}$ versus $\ln [6]$ displaying the first order dependence on dinickel catalyst concentration. All polymerizations were performed at $80{ }^{\circ} \mathrm{C}$ and an initial $\mathrm{CO}_{2}$ pressure of 20.7 bar.

Figure S25. Kinetic plots of $\ln \left([\mathrm{CHO}]_{0} /[\mathrm{CHO}]_{t}\right)$ versus time for $\mathrm{CO}_{2}$-copolymerization of $\mathrm{CHO}$ by dinickel complex 6 at an initial $\mathrm{CO}_{2}$ pressure of 20.7 bar and a catalyst loading of $0.01 \mathrm{~mol} \%$ with various temperatures $\left(100,105,110,120\right.$ and $\left.130{ }^{\circ} \mathrm{C}\right)$.

Table S1. Selected Bond Lengths and Angles of Nickel Complexes 1-11 and $A^{35}$ Table S2. Coupling of $\mathrm{CO}_{2}$ and Terminal Epoxides Catalyzed by Dinickel Complex 6 Table S3. Copolymerization of $\mathrm{CO}_{2}$ and CHO Mediated by Dinickel Complex 6 Under Different Initial $\mathrm{CO}_{2}$ Pressures for $4 \mathrm{~h}$

Table S4. Kinetic Studies of $\mathrm{CO}_{2} / \mathrm{CHO}$ Copolymerization Mediated by Dinickel Complex 6 at Different Monomer-to-Catalyst Ratio $\left(5000,6000,7000,8000\right.$ and 9000) ${ }^{a}$ Table S5. Kinetic Parameters for $\mathrm{CO}_{2} / \mathrm{CHO}$ Copolymerization Mediated by Dinickel Complex 6 at Various Catalyst Concentrations

Table S6. Kinetic Studies of $\mathrm{CO}_{2} / \mathrm{CHO}$ Copolymerization Mediated by Dinickel Complex 6 at Different Temperatures $\left(100,105,110,120 \text { and } 130{ }^{\circ} \mathrm{C}\right)^{a}$

Table S7. Kinetic Parameters for $\mathrm{CO}_{2} / \mathrm{CHO}$ Copolymerization Mediated by Dinickel Complex 6 at Various Temperatures

Table S8. Crystallographic Data of Complexes 1-11 


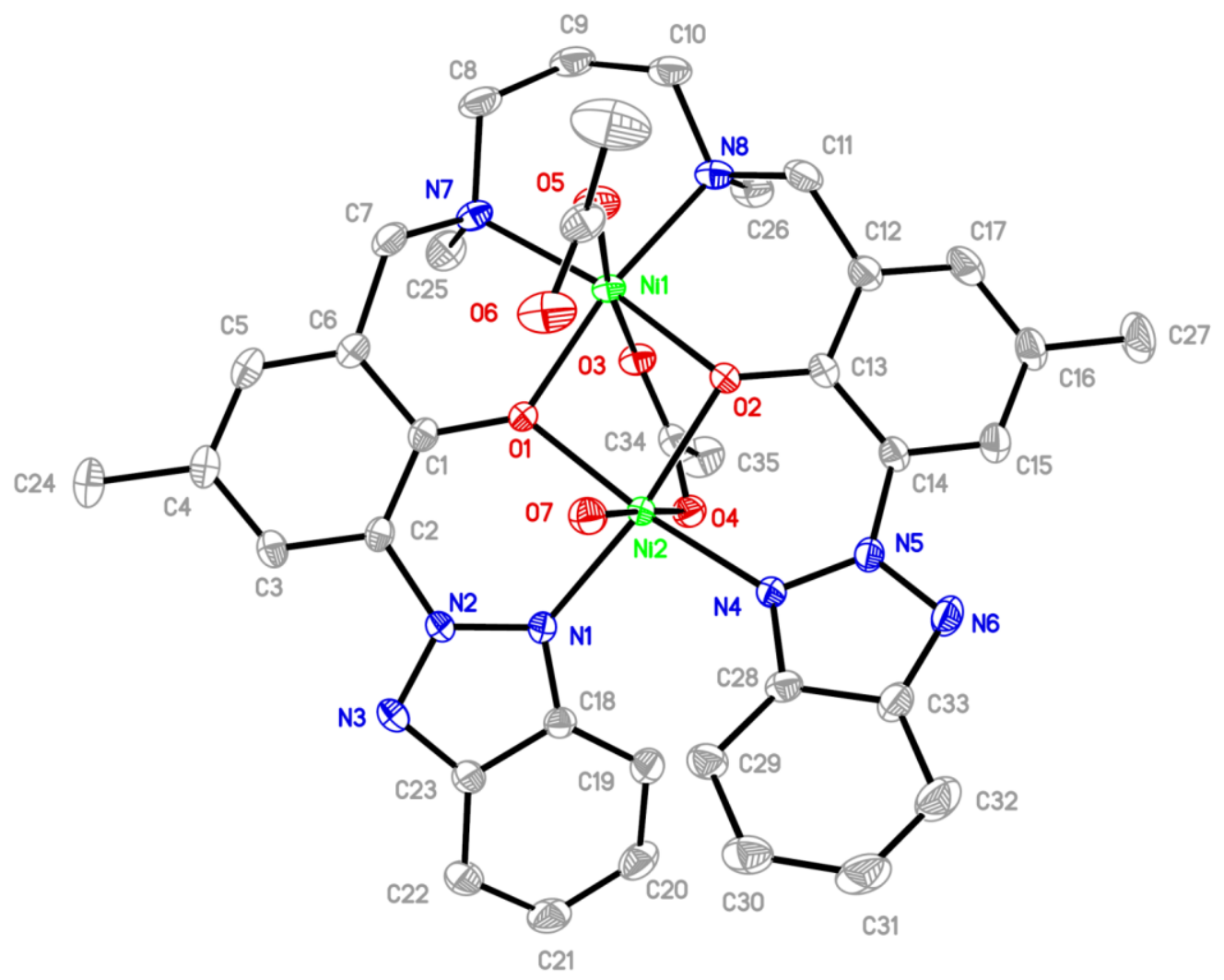

Figure S1. ORTEP drawing of complex 1 with probability ellipsoids drawn at $30 \%$ level. Hydrogen atoms are omitted for clarity. Selected bond lengths $(\AA)$ and angles (deg): $\mathrm{Ni}(1)-\mathrm{O}(1)$ 2.059(2), Ni(1)-O(5) 2.062(2), Ni(1)-O(3) 2.091(2), Ni(1)-O(2) 2.132(2), $\mathrm{Ni}(1)-\mathrm{N}(7)$ 2.140(3), Ni(1)-N(8) 2.172(3), Ni(2)-O(1) 1.998(2), Ni(2)-O(4) 2.048(2), $\mathrm{Ni}(2)-\mathrm{O}(2) \quad 2.056(2), \mathrm{Ni}(2)-\mathrm{N}(4) \quad 2.072(2), \mathrm{Ni}(2)-\mathrm{N}(1) \quad 2.081(3), \mathrm{Ni}(2)-\mathrm{O}(7) \quad 2.145(2)$, $\mathrm{O}(1)-\mathrm{Ni}(1)-\mathrm{O}(5) \quad$ 101.67(9), $\quad \mathrm{O}(1)-\mathrm{Ni}(1)-\mathrm{O}(3) \quad 84.29(9), \quad \mathrm{O}(5)-\mathrm{Ni}(1)-\mathrm{O}(3) \quad$ 172.80(9), $\mathrm{O}(1)-\mathrm{Ni}(1)-\mathrm{O}(2) \quad 81.43(9), \quad \mathrm{O}(5)-\mathrm{Ni}(1)-\mathrm{O}(2) \quad 86.40(9), \quad \mathrm{O}(3)-\mathrm{Ni}(1)-\mathrm{O}(2) \quad$ 90.55(8), $\mathrm{O}(1)-\mathrm{Ni}(1)-\mathrm{N}(7) \quad 88.38(10), \quad \mathrm{O}(5)-\mathrm{Ni}(1)-\mathrm{N}(7) \quad 87.41(10), \quad \mathrm{O}(3)-\mathrm{Ni}(1)-\mathrm{N}(7) \quad$ 96.86(10), $\mathrm{O}(2)-\mathrm{Ni}(1)-\mathrm{N}(7) \quad 166.76(8), \quad \mathrm{O}(1)-\mathrm{Ni}(1)-\mathrm{N}(8) \quad 169.79(9), \quad \mathrm{O}(5)-\mathrm{Ni}(1)-\mathrm{N}(8) \quad 86.44(10)$, $\mathrm{O}(3)-\mathrm{Ni}(1)-\mathrm{N}(8) \quad 87.21(10), \quad \mathrm{O}(2)-\mathrm{Ni}(1)-\mathrm{N}(8) \quad 93.02(10), \quad \mathrm{N}(7)-\mathrm{Ni}(1)-\mathrm{N}(8) \quad$ 98.26(11), $\mathrm{O}(1)-\mathrm{Ni}(2)-\mathrm{O}(4) \quad 97.63(9), \quad \mathrm{O}(1)-\mathrm{Ni}(2)-\mathrm{O}(2) \quad 84.81(9), \quad \mathrm{O}(4)-\mathrm{Ni}(2)-\mathrm{O}(2) \quad 88.89(9)$, $\mathrm{O}(1)-\mathrm{Ni}(2)-\mathrm{N}(4) \quad$ 166.02(9), $\quad \mathrm{O}(4)-\mathrm{Ni}(2)-\mathrm{N}(4) \quad 93.33(10), \quad \mathrm{O}(2)-\mathrm{Ni}(2)-\mathrm{N}(4) \quad 86.77(10)$, $\mathrm{O}(1)-\mathrm{Ni}(2)-\mathrm{N}(1) \quad 86.54(9), \quad \mathrm{O}(4)-\mathrm{Ni}(2)-\mathrm{N}(1) \quad 87.89(10), \quad \mathrm{O}(2)-\mathrm{Ni}(2)-\mathrm{N}(1) \quad 170.29(8)$, $\mathrm{N}(4)-\mathrm{Ni}(2)-\mathrm{N}(1) \quad 102.56(10), \quad \mathrm{O}(1)-\mathrm{Ni}(2)-\mathrm{O}(7) \quad 83.90(9), \quad \mathrm{O}(4)-\mathrm{Ni}(2)-\mathrm{O}(7) \quad$ 178.24(8), $\mathrm{O}(2)-\mathrm{Ni}(2)-\mathrm{O}(7)$ 92.10(9), N(4)-Ni(2)-O(7) 85.27(10), N(1)-Ni(2)-O(7) 91.36(10). 


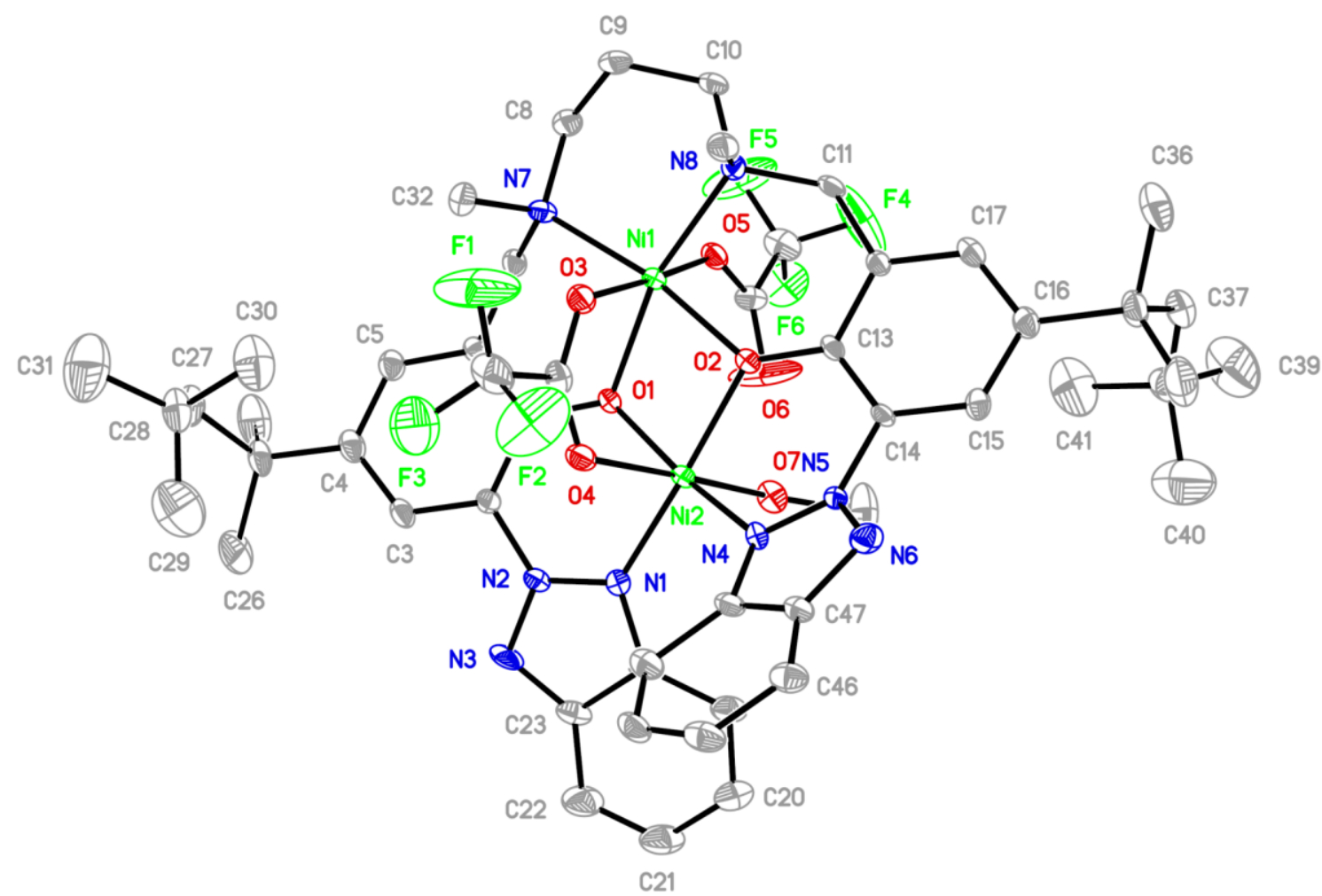

Figure S2. ORTEP drawing of complex 2 with probability ellipsoids drawn at $40 \%$ level. Hydrogen atoms are omitted for clarity. Selected bond lengths $(\AA)$ and angles (deg): $\mathrm{Ni}(1)-\mathrm{O}(2)$ 2.028(5), $\mathrm{Ni}(1)-\mathrm{O}(5)$ 2.070(5), $\mathrm{Ni}(1)-\mathrm{O}(3)$ 2.103(5), $\mathrm{Ni}(1)-\mathrm{O}(1)$ 2.106(5), $\mathrm{Ni}(1)-\mathrm{N}(7)$ 2.105(6), $\mathrm{Ni}(1)-\mathrm{N}(8)$ 2.118(6), Ni(2)-O(2) 1.981(5), Ni(2)-O(1) 2.015(5), $\mathrm{Ni}(2)-\mathrm{N}(1)$ 2.022(6), $\mathrm{Ni}(2)-\mathrm{N}(4)$ 2.052(6), Ni(2)-O(4) 2.065(5), $\mathrm{Ni}(2)-\mathrm{O}(7) \quad 2.100(5)$, $\mathrm{O}(2)-\mathrm{Ni}(1)-\mathrm{O}(5) \quad 100.25(19), \quad \mathrm{O}(2)-\mathrm{Ni}(1)-\mathrm{O}(3) \quad 82.97(19), \quad \mathrm{O}(5)-\mathrm{Ni}(1)-\mathrm{O}(3) \quad 176.8(2)$, $\mathrm{O}(2)-\mathrm{Ni}(1)-\mathrm{O}(1) \quad 81.16(18), \quad \mathrm{O}(5)-\mathrm{Ni}(1)-\mathrm{O}(1) \quad 89.25(19), \quad \mathrm{O}(3)-\mathrm{Ni}(1)-\mathrm{O}(1) \quad 91.10(18)$, $\mathrm{O}(2)-\mathrm{Ni}(1)-\mathrm{N}(7) \quad 169.0(2), \quad \mathrm{O}(5)-\mathrm{Ni}(1)-\mathrm{N}(7) \quad 88.6(2), \quad \mathrm{O}(3)-\mathrm{Ni}(1)-\mathrm{N}(7) \quad 88.2(2)$, $\mathrm{O}(1)-\mathrm{Ni}(1)-\mathrm{N}(7)$ 92.6(2), O(2)-Ni(1)-N(8) 86.9(2), O(5)-Ni(1)-N(8) 84.6(2), O(3)-Ni(1)-N(8) 95.8(2), O(1)-Ni(1)-N(8) 165.4(2), N(7)-Ni(1)-N(8) 100.5(2), O(2)-Ni(2)-O(1) 84.61(19), $\mathrm{O}(2)-\mathrm{Ni}(2)-\mathrm{N}(1) \quad 169.9(2), \quad \mathrm{O}(1)-\mathrm{Ni}(2)-\mathrm{N}(1) \quad 87.3(2), \quad \mathrm{O}(2)-\mathrm{Ni}(2)-\mathrm{N}(4) \quad 86.9(2)$, $\mathrm{O}(1)-\mathrm{Ni}(2)-\mathrm{N}(4) \quad 167.1(2), \quad \mathrm{N}(1)-\mathrm{Ni}(2)-\mathrm{N}(4) \quad 102.0(2), \quad \mathrm{O}(2)-\mathrm{Ni}(2)-\mathrm{O}(4) \quad 94.2(2)$, $\mathrm{O}(1)-\mathrm{Ni}(2)-\mathrm{O}(4)$ 87.1(2), N(1)-Ni(2)-O(4) 91.3(2), N(4)-Ni(2)-O(4) 83.8(2), O(2)-Ni(2)-O(7) 88.8(2), O(1)-Ni(2)-O(7) 89.4(2), N(1)-Ni(2)-O(7) 85.1(2), N(4)-Ni(2)-O(7) 100.1(2), $\mathrm{O}(4)-\mathrm{Ni}(2)-\mathrm{O}(7)$ 175.2(2). 


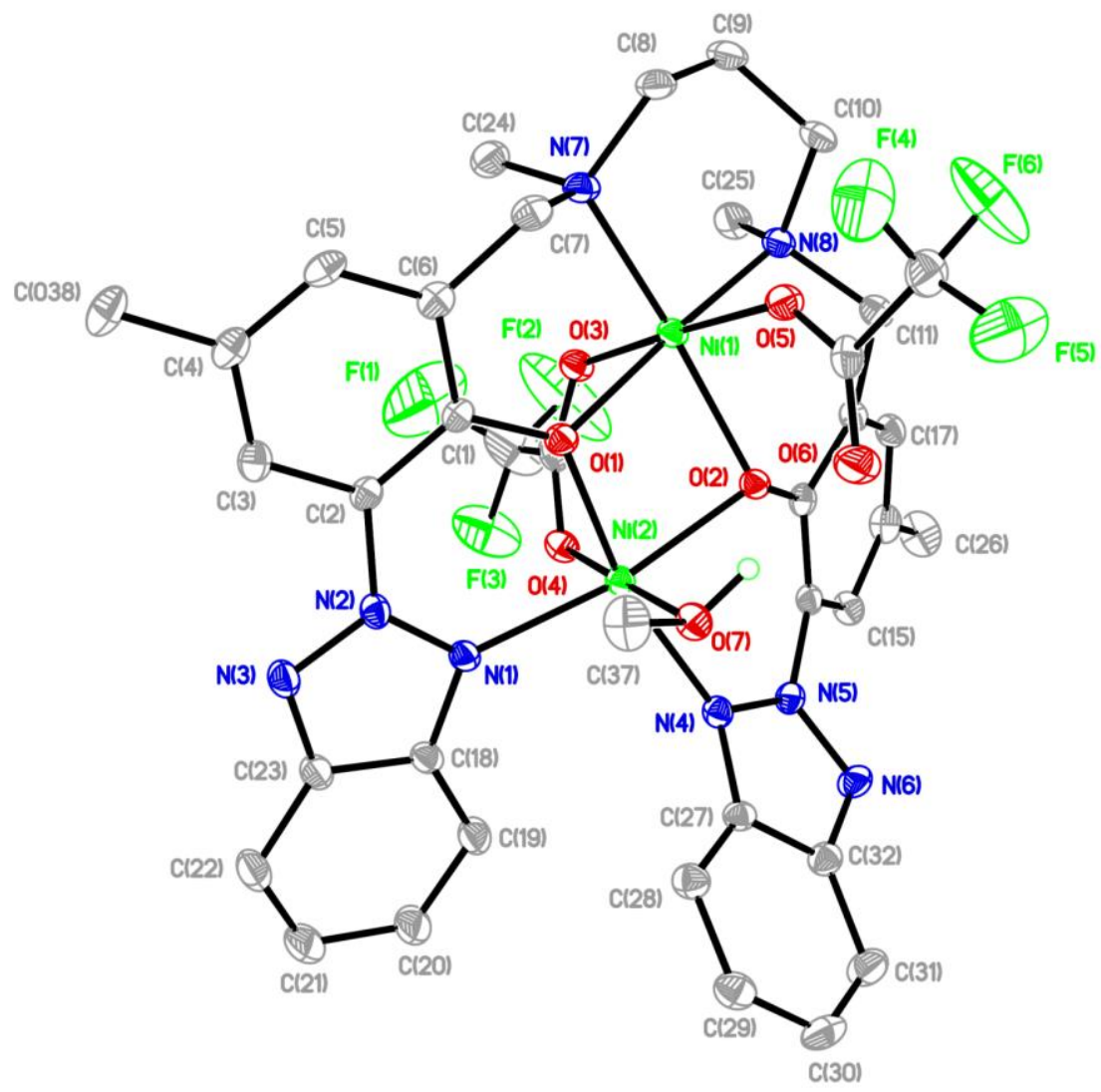

Figure S3. ORTEP drawing of complex 3 with probability ellipsoids drawn at $30 \%$ level. Hydrogen atoms (except that of the coordinated $\mathrm{MeOH}$ ) are omitted for clarity. Selected bond lengths $(\AA)$ and angles (deg): Ni(1)-O(3) 2.069(3), Ni(1)-O(1) 2.080(3), Ni(1)-O(2) 2.099(3), $\mathrm{Ni}(1)-\mathrm{O}(5) \quad 2.103(3), \quad \mathrm{Ni}(1)-\mathrm{N}(7) \quad 2.122(4), \quad \mathrm{Ni}(1)-\mathrm{N}(8) \quad 2.135(4), \quad \mathrm{Ni}(2)-\mathrm{O}(1)$ 1.993(3), $\mathrm{Ni}(2)-\mathrm{O}(2) \quad 2.056(3), \quad \mathrm{Ni}(2)-\mathrm{O}(4) \quad 2.059(3), \quad \mathrm{Ni}(2)-\mathrm{N}(4) \quad 2.080(4), \quad \mathrm{Ni}(2)-\mathrm{N}(1)$ 2.081(4), Ni(2)-O(7) 2.099(3), O(3)-Ni(1)-O(1) 85.09(12), O(3)-Ni(1)-O(2) 89.63(12), $\mathrm{O}(1)-\mathrm{Ni}(1)-\mathrm{O}(2) \quad 81.14(11), \quad \mathrm{O}(3)-\mathrm{Ni}(1)-\mathrm{O}(5) \quad$ 177.28(13), $\mathrm{O}(1)-\mathrm{Ni}(1)-\mathrm{O}(5) \quad$ 95.64(13), $\mathrm{O}(2)-\mathrm{Ni}(1)-\mathrm{O}(5) \quad 87.90(12), \quad \mathrm{O}(3)-\mathrm{Ni}(1)-\mathrm{N}(7) \quad 96.08(14), \quad \mathrm{O}(1)-\mathrm{Ni}(1)-\mathrm{N}(7) \quad 90.72(13)$, $\mathrm{O}(2)-\mathrm{Ni}(1)-\mathrm{N}(7) \quad$ 169.65(13), $\quad \mathrm{O}(5)-\mathrm{Ni}(1)-\mathrm{N}(7) \quad 86.54(14), \quad \mathrm{O}(3)-\mathrm{Ni}(1)-\mathrm{N}(8) \quad 88.92(13)$, $\mathrm{O}(1)-\mathrm{Ni}(1)-\mathrm{N}(8) \quad$ 172.59(13), $\quad \mathrm{O}(2)-\mathrm{Ni}(1)-\mathrm{N}(8) \quad 94.48(13), \quad \mathrm{O}(5)-\mathrm{Ni}(1)-\mathrm{N}(8) \quad 90.14(14)$, $\mathrm{N}(7)-\mathrm{Ni}(1)-\mathrm{N}(8) \quad 94.26(15), \quad \mathrm{O}(1)-\mathrm{Ni}(2)-\mathrm{O}(2) \quad 84.31(12), \quad \mathrm{O}(1)-\mathrm{Ni}(2)-\mathrm{O}(4) \quad 95.82(13)$, $\mathrm{O}(2)-\mathrm{Ni}(2)-\mathrm{O}(4) \quad 87.53(12), \quad \mathrm{O}(1)-\mathrm{Ni}(2)-\mathrm{N}(4) \quad$ 164.61(14), $\mathrm{O}(2)-\mathrm{Ni}(2)-\mathrm{N}(4) \quad 85.29(13)$, $\mathrm{O}(4)-\mathrm{Ni}(2)-\mathrm{N}(4) \quad 95.04(14), \quad \mathrm{O}(1)-\mathrm{Ni}(2)-\mathrm{N}(1) \quad 88.74(13), \quad \mathrm{O}(2)-\mathrm{Ni}(2)-\mathrm{N}(1) \quad$ 169.70(14), $\mathrm{O}(4)-\mathrm{Ni}(2)-\mathrm{N}(1) \quad 85.62(14), \quad \mathrm{N}(4)-\mathrm{Ni}(2)-\mathrm{N}(1) \quad$ 102.95(14), $\quad \mathrm{O}(1)-\mathrm{Ni}(2)-\mathrm{O}(7) \quad 86.25(13)$, $\mathrm{O}(2)-\mathrm{Ni}(2)-\mathrm{O}(7) \quad 90.61(12), \quad \mathrm{O}(4)-\mathrm{Ni}(2)-\mathrm{O}(7) \quad$ 177.06(13), $\quad \mathrm{N}(4)-\mathrm{Ni}(2)-\mathrm{O}(7) \quad 82.54(14)$, $\mathrm{N}(1)-\mathrm{Ni}(2)-\mathrm{O}(7) 96.53(14)$. 


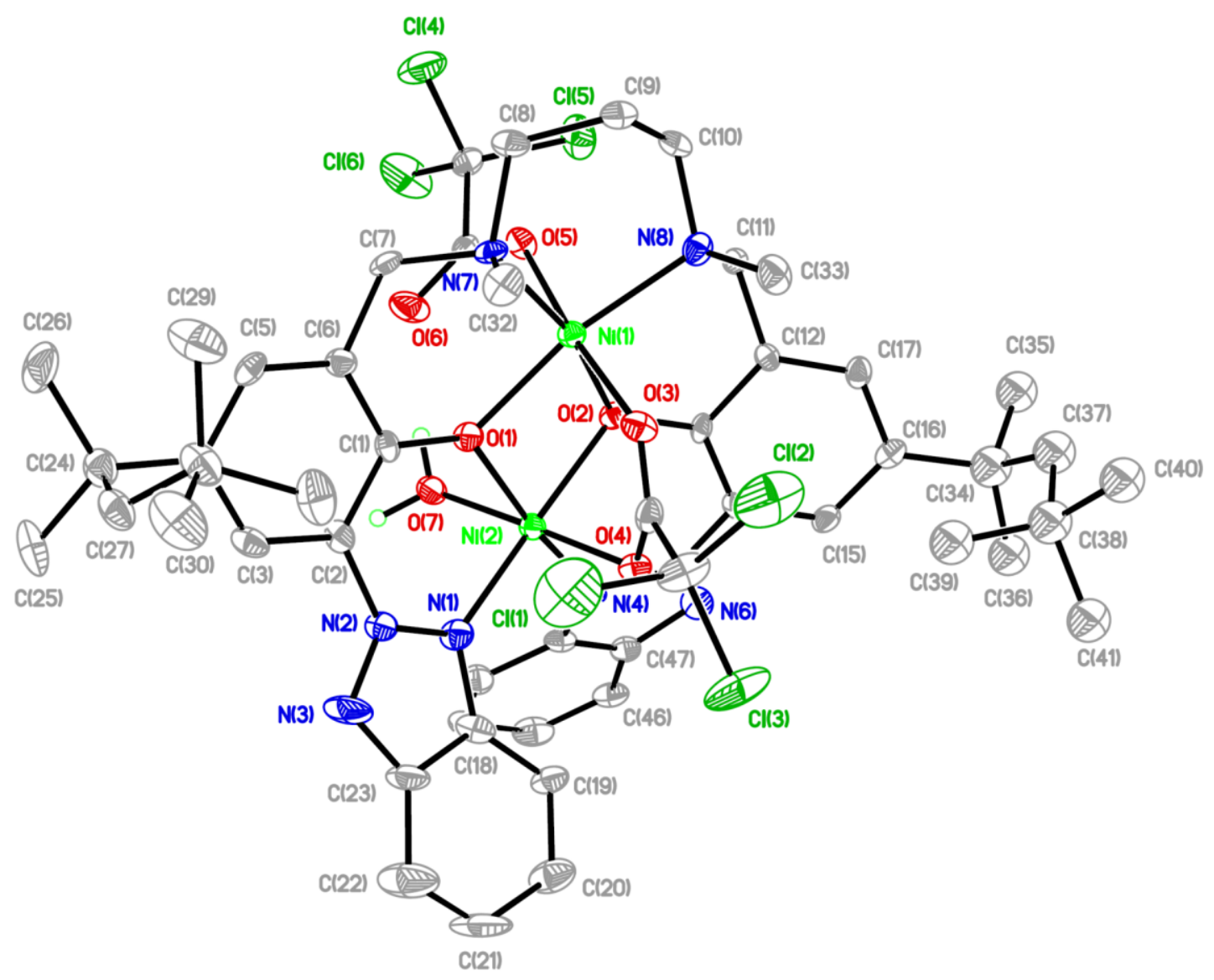

Figure S4. ORTEP drawing of complex 4 with probability ellipsoids drawn at 50\% level. Hydrogen atoms (except that of the coordinated water) are omitted for clarity. Selected bond lengths $(\AA)$ and angles (deg): Ni(1)-O(1) 2.047(5), Ni(1)-O(5) 2.067(5), Ni(1)-O(3) 2.081(5), $\mathrm{Ni}(1)-\mathrm{O}(2) \quad 2.095(5), \mathrm{Ni}(1)-\mathrm{N}(7) \quad 2.125(5), \mathrm{Ni}(1)-\mathrm{N}(8) \quad 2.130(6), \mathrm{Ni}(2)-\mathrm{O}(1)$ 1.990(5), $\mathrm{Ni}(2)-\mathrm{N}(4)$ 2.038(6), Ni(2)-O(2) 2.039(5), Ni(2)-N(1) 2.068(6), Ni(2)-O(7) 2.087(5), $\mathrm{Ni}(2)-\mathrm{O}(4) \quad 2.102(5), \quad \mathrm{O}(1)-\mathrm{Ni}(1)-\mathrm{O}(5) \quad$ 102.61(19), $\quad \mathrm{O}(1)-\mathrm{Ni}(1)-\mathrm{O}(3) \quad 82.34(19)$, $\mathrm{O}(5)-\mathrm{Ni}(1)-\mathrm{O}(3) \quad$ 174.33(19), $\quad \mathrm{O}(1)-\mathrm{Ni}(1)-\mathrm{O}(2) \quad 80.40(18), \quad \mathrm{O}(5)-\mathrm{Ni}(1)-\mathrm{O}(2) \quad 85.85(19)$, $\mathrm{O}(3)-\mathrm{Ni}(1)-\mathrm{O}(2) \quad 92.32(19), \quad \mathrm{O}(1)-\mathrm{Ni}(1)-\mathrm{N}(7) \quad 89.0(2), \quad \mathrm{O}(5)-\mathrm{Ni}(1)-\mathrm{N}(7) \quad 86.9(2)$, $\mathrm{O}(3)-\mathrm{Ni}(1)-\mathrm{N}(7) \quad 96.0(2), \quad \mathrm{O}(2)-\mathrm{Ni}(1)-\mathrm{N}(7) \quad 165.5(2), \quad \mathrm{O}(1)-\mathrm{Ni}(1)-\mathrm{N}(8) \quad 169.0(2)$, $\mathrm{O}(5)-\mathrm{Ni}(1)-\mathrm{N}(8)$ 86.9(2), O(3)-Ni(1)-N(8) 87.9(2), O(2)-Ni(1)-N(8) 95.0(2), N(7)-Ni(1)-N(8) 97.1(2), O(1)-Ni(2)-N(4) 167.1(2), O(1)-Ni(2)-O(2) 83.14(18), N(4)-Ni(2)-O(2) 87.8(2), $\mathrm{O}(1)-\mathrm{Ni}(2)-\mathrm{N}(1) \quad 87.9(2), \quad \mathrm{N}(4)-\mathrm{Ni}(2)-\mathrm{N}(1) \quad 102.7(2), \quad \mathrm{O}(2)-\mathrm{Ni}(2)-\mathrm{N}(1) \quad 165.5(2)$, $\mathrm{O}(1)-\mathrm{Ni}(2)-\mathrm{O}(7) \quad 85.5(2), \quad \mathrm{N}(4)-\mathrm{Ni}(2)-\mathrm{O}(7) \quad 86.2(2), \quad \mathrm{O}(2)-\mathrm{Ni}(2)-\mathrm{O}(7) \quad$ 94.68(19), $\mathrm{N}(1)-\mathrm{Ni}(2)-\mathrm{O}(7) \quad 96.0(2), \quad \mathrm{O}(1)-\mathrm{Ni}(2)-\mathrm{O}(4) \quad 93.24(19), \quad \mathrm{N}(4)-\mathrm{Ni}(2)-\mathrm{O}(4) \quad 95.4(2)$, $\mathrm{O}(2)-\mathrm{Ni}(2)-\mathrm{O}(4)$ 87.22(19), N(1)-Ni(2)-O(4) 81.9(2), O(7)-Ni(2)-O(4) 177.57(19). 


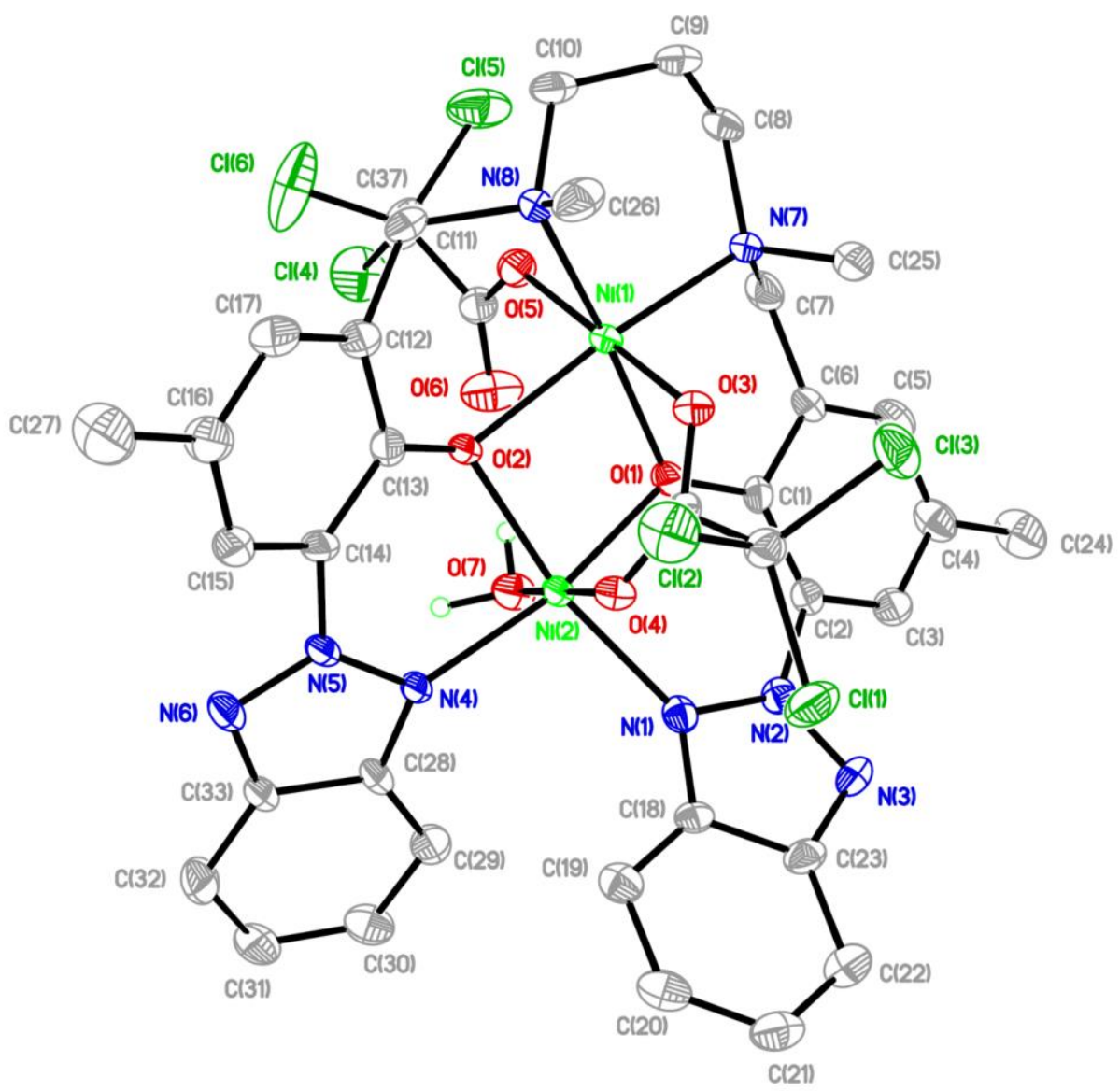

Figure S5. ORTEP drawing of complex 5 with probability ellipsoids drawn at 50\% level. Hydrogen atoms (except that of the coordinated water) are omitted for clarity. Selected bond lengths ( $\AA$ ) and angles (deg): Ni(1)-O(1) 2.039(4), Ni(1)-O(5) 2.091(4), Ni(1)-O(3) 2.100(4), $\mathrm{Ni}(1)-\mathrm{O}(2)$ 2.104(4), $\mathrm{Ni}(1)-\mathrm{N}(7)$ 2.119(4), $\mathrm{Ni}(1)-\mathrm{N}(8)$ 2.125(5), $\mathrm{Ni}(2)-\mathrm{O}(1)$ 1.982(4), $\mathrm{Ni}(2)-\mathrm{N}(4)$ 2.035(4), $\mathrm{Ni}(2)-\mathrm{O}(2)$ 2.043(4), Ni(2)-N(1) 2.069(4), Ni(2)-O(4) 2.083(4), $\mathrm{Ni}(2)-\mathrm{O}(7) \quad 2.125(4), \quad \mathrm{O}(1)-\mathrm{Ni}(1)-\mathrm{O}(5) \quad 101.72(15), \quad \mathrm{O}(1)-\mathrm{Ni}(1)-\mathrm{O}(3) \quad 82.68(15)$, $\mathrm{O}(5)-\mathrm{Ni}(1)-\mathrm{O}(3) \quad$ 175.11(15), $\quad \mathrm{O}(1)-\mathrm{Ni}(1)-\mathrm{O}(2) \quad 79.98(14), \quad \mathrm{O}(5)-\mathrm{Ni}(1)-\mathrm{O}(2) \quad 85.27(15)$, $\mathrm{O}(3)-\mathrm{Ni}(1)-\mathrm{O}(2) \quad 93.49(14), \quad \mathrm{O}(1)-\mathrm{Ni}(1)-\mathrm{N}(7) \quad 89.25(16), \quad \mathrm{O}(5)-\mathrm{Ni}(1)-\mathrm{N}(7) \quad 86.88(16)$, $\mathrm{O}(3)-\mathrm{Ni}(1)-\mathrm{N}(7) \quad 95.35(16), \quad \mathrm{O}(2)-\mathrm{Ni}(1)-\mathrm{N}(7) \quad$ 165.08(16), $\mathrm{O}(1)-\mathrm{Ni}(1)-\mathrm{N}(8) \quad$ 168.82(16), $\mathrm{O}(5)-\mathrm{Ni}(1)-\mathrm{N}(8) \quad 87.54(17), \quad \mathrm{O}(3)-\mathrm{Ni}(1)-\mathrm{N}(8) \quad 87.85(16), \quad \mathrm{O}(2)-\mathrm{Ni}(1)-\mathrm{N}(8) \quad 94.74(15)$, $\mathrm{N}(7)-\mathrm{Ni}(1)-\mathrm{N}(8) \quad 97.58(17), \quad \mathrm{O}(1)-\mathrm{Ni}(2)-\mathrm{N}(4) \quad 166.40(17), \quad \mathrm{O}(1)-\mathrm{Ni}(2)-\mathrm{O}(2) \quad 82.81(15)$, $\mathrm{N}(4)-\mathrm{Ni}(2)-\mathrm{O}(2) \quad 88.56(16), \quad \mathrm{O}(1)-\mathrm{Ni}(2)-\mathrm{N}(1) \quad 89.06(16), \quad \mathrm{N}(4)-\mathrm{Ni}(2)-\mathrm{N}(1) \quad$ 101.39(17), $\mathrm{O}(2)-\mathrm{Ni}(2)-\mathrm{N}(1) \quad$ 165.88(16), $\mathrm{O}(1)-\mathrm{Ni}(2)-\mathrm{O}(4) \quad 94.81(15), \quad \mathrm{N}(4)-\mathrm{Ni}(2)-\mathrm{O}(4) \quad 95.15(17)$, $\mathrm{O}(2)-\mathrm{Ni}(2)-\mathrm{O}(4) \quad 86.61(15), \quad \mathrm{N}(1)-\mathrm{Ni}(2)-\mathrm{O}(4) \quad 82.58(16), \quad \mathrm{O}(1)-\mathrm{Ni}(2)-\mathrm{O}(7) \quad 83.52(16)$, $\mathrm{N}(4)-\mathrm{Ni}(2)-\mathrm{O}(7) \quad 86.90(18), \quad \mathrm{O}(2)-\mathrm{Ni}(2)-\mathrm{O}(7) \quad 95.60(16), \quad \mathrm{N}(1)-\mathrm{Ni}(2)-\mathrm{O}(7) \quad 94.93(17)$, $\mathrm{O}(4)-\mathrm{Ni}(2)-\mathrm{O}(7)$ 177.03(15). 


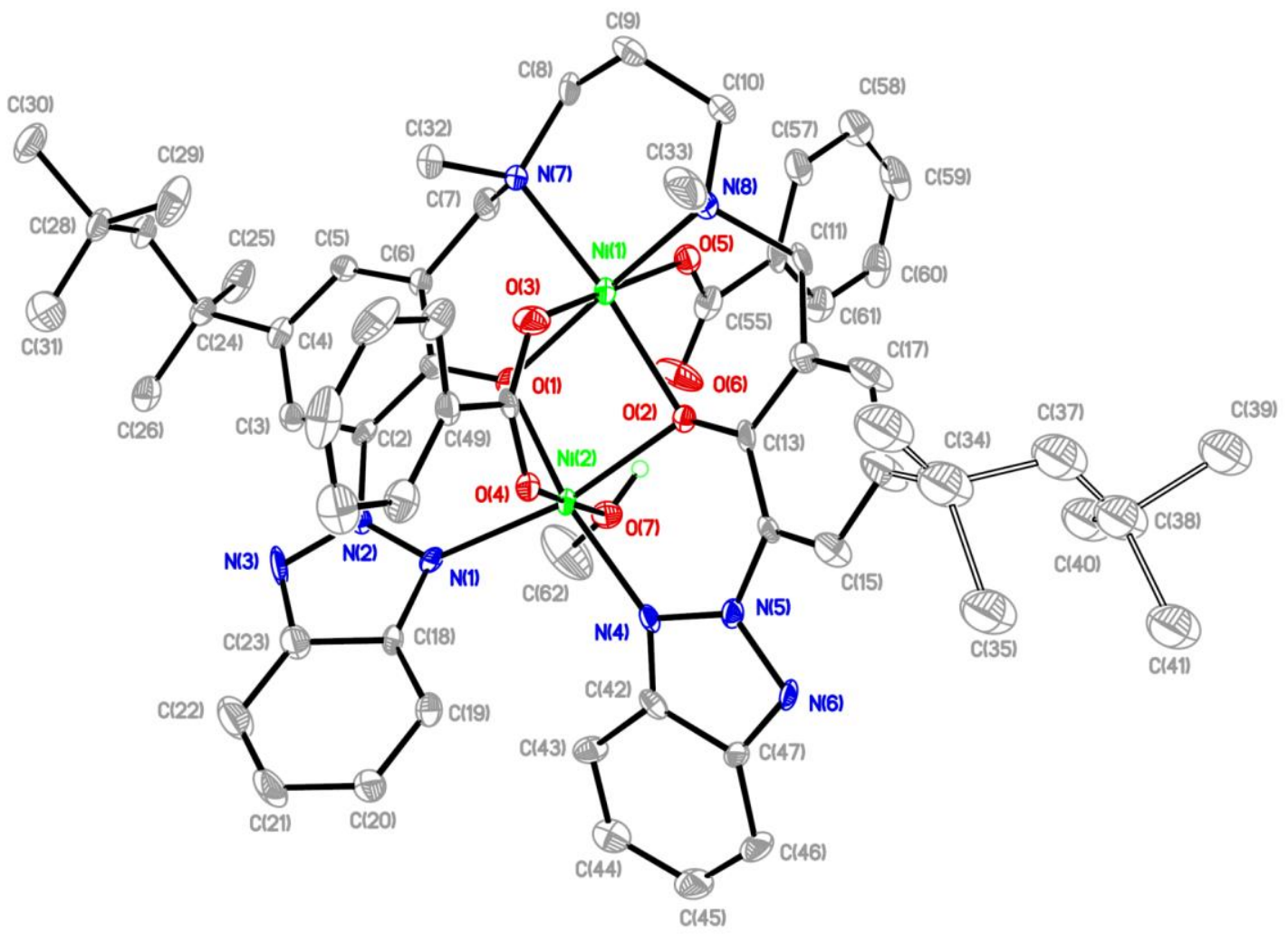

Figure S6. ORTEP drawing of complex 6 with probability ellipsoids drawn at $30 \%$ level. Hydrogen atoms (except that of the coordinated $\mathrm{MeOH}$ ) are omitted for clarity. Selected bond lengths $(\AA)$ and angles (deg): Ni(1)-O(1) 2.043(7), Ni(1)-O(2) 2.103(7), Ni(1)-O(3) 2.070(9), $\mathrm{Ni}(1)-\mathrm{O}(5) \quad 2.093(9), \quad \mathrm{Ni}(1)-\mathrm{N}(7) \quad 2.144(9), \quad \mathrm{Ni}(1)-\mathrm{N}(8) \quad 2.136(9), \quad \mathrm{Ni}(2)-\mathrm{O}(1)$ 2.002(7), $\mathrm{Ni}(2)-\mathrm{O}(2) \quad 2.052(7), \quad \mathrm{Ni}(2)-\mathrm{O}(4) \quad 2.007(8), \quad \mathrm{Ni}(2)-\mathrm{O}(7) \quad 2.087(8), \quad \mathrm{Ni}(2)-\mathrm{N}(1)$ 2.085(8), $\quad \mathrm{Ni}(2)-\mathrm{N}(4) \quad 2.042(9), \quad \mathrm{O}(1)-\mathrm{Ni}(1)-\mathrm{O}(2) \quad 82.0(3), \quad \mathrm{O}(1)-\mathrm{Ni}(1)-\mathrm{O}(3) \quad 83.4(3)$, $\mathrm{O}(1)-\mathrm{Ni}(1)-\mathrm{O}(5) \quad 102.8(3), \quad \mathrm{O}(1)-\mathrm{Ni}(1)-\mathrm{N}(7) \quad 87.4(3), \quad \mathrm{O}(1)-\mathrm{Ni}(1)-\mathrm{N}(8) \quad 169.8(3)$, $\mathrm{O}(2)-\mathrm{Ni}(1)-\mathrm{N}(7) \quad$ 166.1(3), $\quad \mathrm{O}(2)-\mathrm{Ni}(1)-\mathrm{N}(8) \quad 94.7(3), \quad \mathrm{O}(3)-\mathrm{Ni}(1)-\mathrm{O}(2) \quad 90.7(3)$, $\mathrm{O}(3)-\mathrm{Ni}(1)-\mathrm{O}(5) \quad$ 173.3(3), $\quad \mathrm{O}(3)-\mathrm{Ni}(1)-\mathrm{N}(7) \quad 97.0(4), \quad \mathrm{O}(3)-\mathrm{Ni}(1)-\mathrm{N}(8) \quad 87.0(3)$, $\mathrm{O}(5)-\mathrm{Ni}(1)-\mathrm{O}(2)$ 87.6(3), O(5)-Ni(1)-N(7) 86.0(3), O(5)-Ni(1)-N(8) 86.6(4), N(8)-Ni(1)-N(7) 97.3(4), O(1)-Ni(2)-O(2) 84.3(3), O(1)-Ni(2)-O(4) 95.3(3), O(1)-Ni(2)-O(7) 85.1(3), $\mathrm{O}(1)-\mathrm{Ni}(2)-\mathrm{N}(1) \quad 86.1(3), \quad \mathrm{O}(1)-\mathrm{Ni}(2)-\mathrm{N}(4) \quad 168.9(3), \quad \mathrm{O}(2)-\mathrm{Ni}(2)-\mathrm{O}(7) \quad 89.7(3)$, $\mathrm{O}(2)-\mathrm{Ni}(2)-\mathrm{N}(1) \quad$ 167.2(3), $\quad \mathrm{O}(4)-\mathrm{Ni}(2)-\mathrm{O}(2) \quad 88.5(3), \quad \mathrm{O}(4)-\mathrm{Ni}(2)-\mathrm{O}(7) \quad$ 178.2(3), $\mathrm{O}(4)-\mathrm{Ni}(2)-\mathrm{N}(1)$ 84.0(3), O(4)-Ni(2)-N(4) 92.0(3), N(1)-Ni(2)-O(7) 97.9(3), N(4)-Ni(2)-O(2) 87.5(3), N(4)-Ni(2)-O(7) 87.4(3), N(4)-Ni(2)-N(1) 103.0(3). 


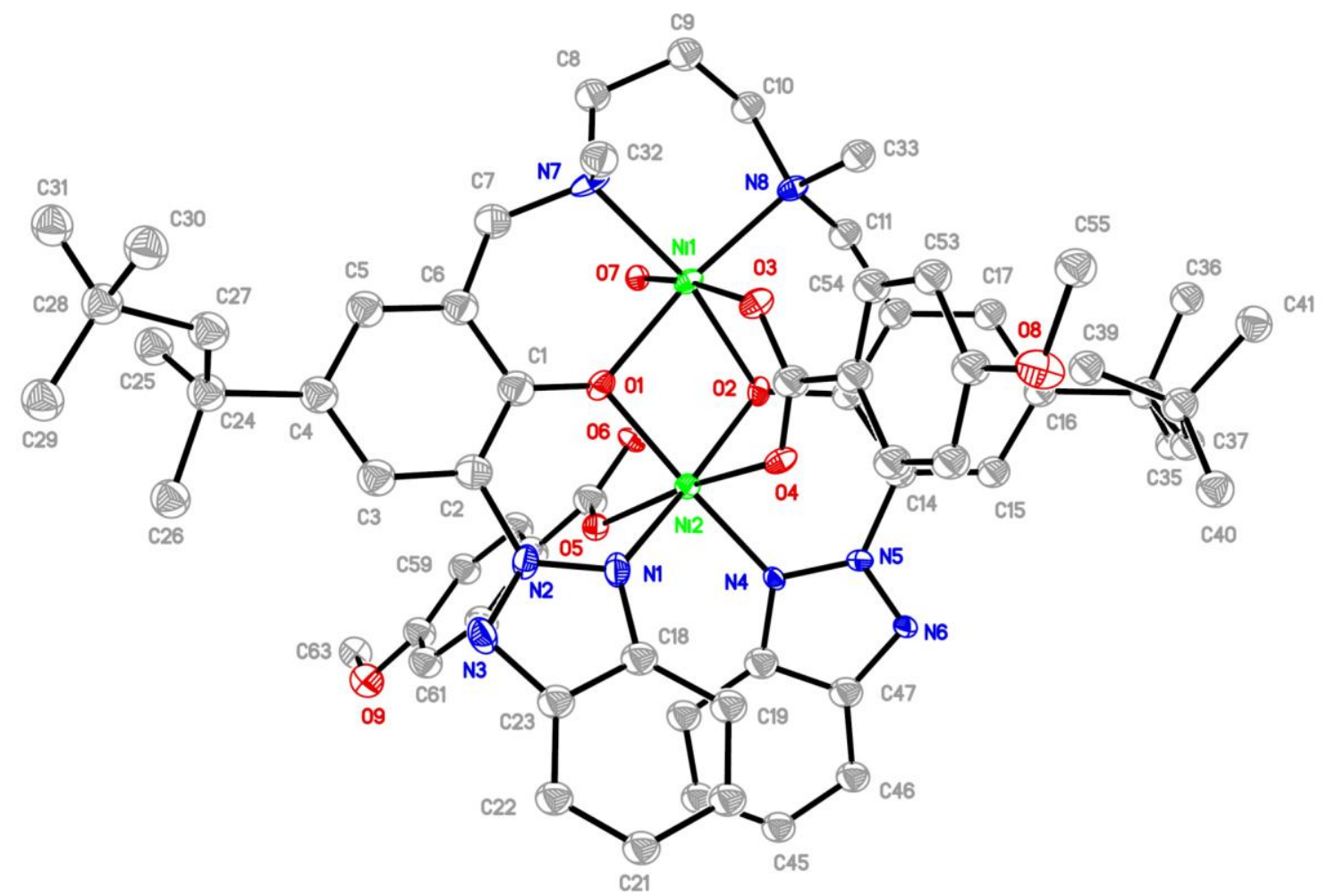

Figure S7. ORTEP drawing of complex $\mathbf{8}$ with probability ellipsoids drawn at $30 \%$ level. Hydrogen atoms are omitted for clarity. Selected bond lengths $(\AA)$ and angles (deg): $\mathrm{Ni}(1)-\mathrm{O}(3)$ 2.042(10), Ni(1)-O(1) 2.052(10), Ni(1)-O(7) 2.100(10), Ni(1)-N(7) 2.118(12), $\mathrm{Ni}(1)-\mathrm{N}(8)$ 2.126(12), Ni(1)-O(2) 2.133(10), Ni(2)-O(1) 1.983(10), Ni(2)-O(2) 2.030(10), $\mathrm{Ni}(2)-\mathrm{N}(4)$ 2.059(12), Ni(2)-O(5) 2.058(10), Ni(2)-N(1) 2.074(13), Ni(2)-O(4) 2.095(10), $\mathrm{O}(3)-\mathrm{Ni}(1)-\mathrm{O}(1) \quad 85.6(4), \quad \mathrm{O}(3)-\mathrm{Ni}(1)-\mathrm{O}(7) \quad$ 170.4(4), $\quad \mathrm{O}(1)-\mathrm{Ni}(1)-\mathrm{O}(7) \quad 93.5(4)$, $\mathrm{O}(3)-\mathrm{Ni}(1)-\mathrm{N}(7)$ 97.4(4), O(1)-Ni(1)-N(7) 88.9(5), O(7)-Ni(1)-N(7) 92.1(4), O(3)-Ni(1)-N(8) 89.0(4), O(1)-Ni(1)-N(8) 172.0(4), O(7)-Ni(1)-N(8) 90.9(4), N(7)-Ni(1)-N(8) 97.7(5), $\mathrm{O}(3)-\mathrm{Ni}(1)-\mathrm{O}(2)$ 91.0(4), O(1)-Ni(1)-O(2) 82.1(4), O(7)-Ni(1)-O(2) 79.5(4), N(7)-Ni(1)-O(2) 167.2(4), N(8)-Ni(1)-O(2) 92.1(4), O(1)-Ni(2)-O(2) 86.5(4), O(1)-Ni(2)-N(4) 169.8(4), $\mathrm{O}(2)-\mathrm{Ni}(2)-\mathrm{N}(4) \quad 86.3(4), \quad \mathrm{O}(1)-\mathrm{Ni}(2)-\mathrm{O}(5) \quad 88.0(4), \quad \mathrm{O}(2)-\mathrm{Ni}(2)-\mathrm{O}(5) \quad$ 103.0(4), $\mathrm{N}(4)-\mathrm{Ni}(2)-\mathrm{O}(5) \quad 86.7(4), \quad \mathrm{O}(1)-\mathrm{Ni}(2)-\mathrm{N}(1) \quad 87.2(5), \quad \mathrm{O}(2)-\mathrm{Ni}(2)-\mathrm{N}(1) \quad$ 167.2(5), $\mathrm{N}(4)-\mathrm{Ni}(2)-\mathrm{N}(1) \quad$ 101.2(5), $\quad \mathrm{O}(5)-\mathrm{Ni}(2)-\mathrm{N}(1) \quad 87.9(4), \quad \mathrm{O}(1)-\mathrm{Ni}(2)-\mathrm{O}(4) \quad$ 94.4(4), $\mathrm{O}(2)-\mathrm{Ni}(2)-\mathrm{O}(4) \quad 85.7(4), \quad \mathrm{N}(4)-\mathrm{Ni}(2)-\mathrm{O}(4) \quad 92.1(4), \quad \mathrm{O}(5)-\mathrm{Ni}(2)-\mathrm{O}(4) \quad 171.1(4)$, $\mathrm{N}(1)-\mathrm{Ni}(2)-\mathrm{O}(4)$ 83.7(4). 


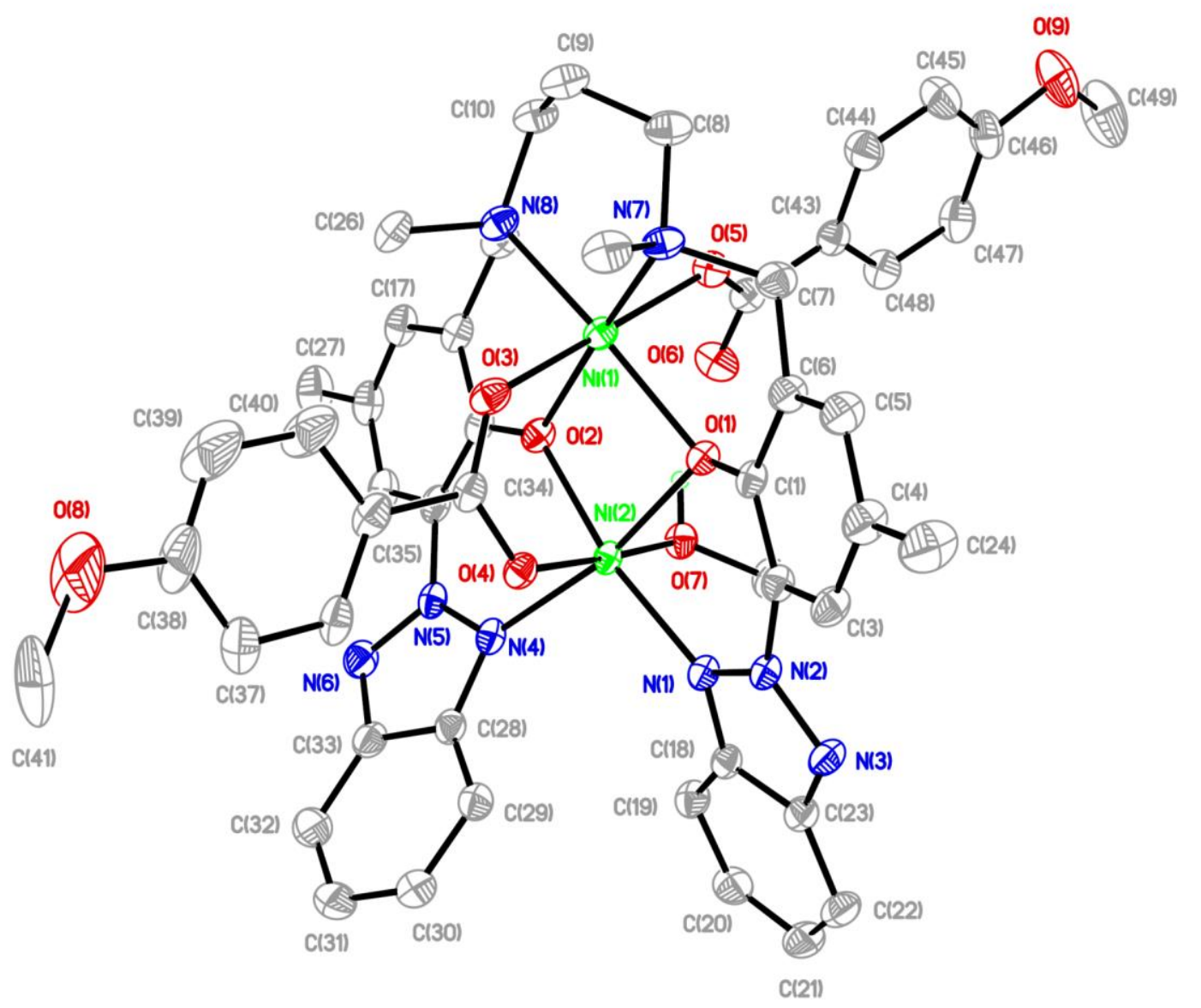

Figure S8. ORTEP drawing of complex 9 with probability ellipsoids drawn at $30 \%$ level. Hydrogen atoms (except that of the coordinated $\mathrm{MeOH}$ ) are omitted for clarity. Selected bond lengths $(\AA)$ and angles (deg): $\mathrm{Ni}(1)-\mathrm{O}(2)$ 2.044(2), Ni(1)-O(5) 2.062(2), Ni(1)-O(3) 2.071(2), $\mathrm{Ni}(1)-\mathrm{O}(1)$ 2.116(2), $\mathrm{Ni}(1)-\mathrm{N}(8)$ 2.130(3), $\mathrm{Ni}(1)-\mathrm{N}(7) \quad 2.128(3), \quad \mathrm{Ni}(2)-\mathrm{O}(2)$ 1.990(2), $\mathrm{Ni}(2)-\mathrm{O}(1)$ 2.034(2), $\mathrm{Ni}(2)-\mathrm{N}(1)$ 2.033(3), $\mathrm{Ni}(2)-\mathrm{O}(4) \quad 2.052(2), \quad \mathrm{Ni}(2)-\mathrm{N}(4)$ 2.075(3), $\mathrm{Ni}(2)-\mathrm{O}(7)$ 2.122(2), O(2)-Ni(1)-O(5) 102.91(9), O(2)-Ni(1)-O(3) 82.09(9), $\mathrm{O}(5)-\mathrm{Ni}(1)-\mathrm{O}(3) \quad$ 174.68(9), $\quad \mathrm{O}(2)-\mathrm{Ni}(1)-\mathrm{O}(1) \quad 81.42(8), \quad \mathrm{O}(5)-\mathrm{Ni}(1)-\mathrm{O}(1) \quad 87.61(9)$, $\mathrm{O}(3)-\mathrm{Ni}(1)-\mathrm{O}(1) \quad 91.41(8), \quad \mathrm{O}(2)-\mathrm{Ni}(1)-\mathrm{N}(8) \quad 88.18(9), \quad \mathrm{O}(5)-\mathrm{Ni}(1)-\mathrm{N}(8) \quad 87.68(10)$, $\mathrm{O}(3)-\mathrm{Ni}(1)-\mathrm{N}(8) \quad 94.31(10), \quad \mathrm{O}(1)-\mathrm{Ni}(1)-\mathrm{N}(8) \quad 167.33(9), \quad \mathrm{O}(2)-\mathrm{Ni}(1)-\mathrm{N}(7) \quad$ 168.43(10), $\mathrm{O}(5)-\mathrm{Ni}(1)-\mathrm{N}(7) \quad 86.69(10), \quad \mathrm{O}(3)-\mathrm{Ni}(1)-\mathrm{N}(7) \quad 88.14(10), \quad \mathrm{O}(1)-\mathrm{Ni}(1)-\mathrm{N}(7) \quad 92.69(9)$, $\mathrm{N}(8)-\mathrm{Ni}(1)-\mathrm{N}(7) \quad 98.77(10), \quad \mathrm{O}(2)-\mathrm{Ni}(2)-\mathrm{O}(1) \quad 84.77(8), \quad \mathrm{O}(2)-\mathrm{Ni}(2)-\mathrm{N}(1) \quad 169.98(10)$, $\mathrm{O}(1)-\mathrm{Ni}(2)-\mathrm{N}(1) \quad 87.88(9), \quad \mathrm{O}(2)-\mathrm{Ni}(2)-\mathrm{O}(4) \quad 94.24(8), \quad \mathrm{O}(1)-\mathrm{Ni}(2)-\mathrm{O}(4) \quad 88.72(8)$, $\mathrm{N}(1)-\mathrm{Ni}(2)-\mathrm{O}(4) \quad 92.40(9), \quad \mathrm{O}(2)-\mathrm{Ni}(2)-\mathrm{N}(4) \quad 86.48(9), \quad \mathrm{O}(1)-\mathrm{Ni}(2)-\mathrm{N}(4) \quad 167.95(9)$, $\mathrm{N}(1)-\mathrm{Ni}(2)-\mathrm{N}(4) \quad$ 101.73(10), $\quad \mathrm{O}(4)-\mathrm{Ni}(2)-\mathrm{N}(4) \quad 83.67(9), \quad \mathrm{O}(2)-\mathrm{Ni}(2)-\mathrm{O}(7) \quad 81.90(9)$, $\mathrm{O}(1)-\mathrm{Ni}(2)-\mathrm{O}(7) \quad 93.31(8), \quad \mathrm{N}(1)-\mathrm{Ni}(2)-\mathrm{O}(7) \quad 91.75(9), \quad \mathrm{O}(4)-\mathrm{Ni}(2)-\mathrm{O}(7) \quad$ 175.45(8), $\mathrm{N}(4)-\mathrm{Ni}(2)-\mathrm{O}(7)$ 93.65(9). 


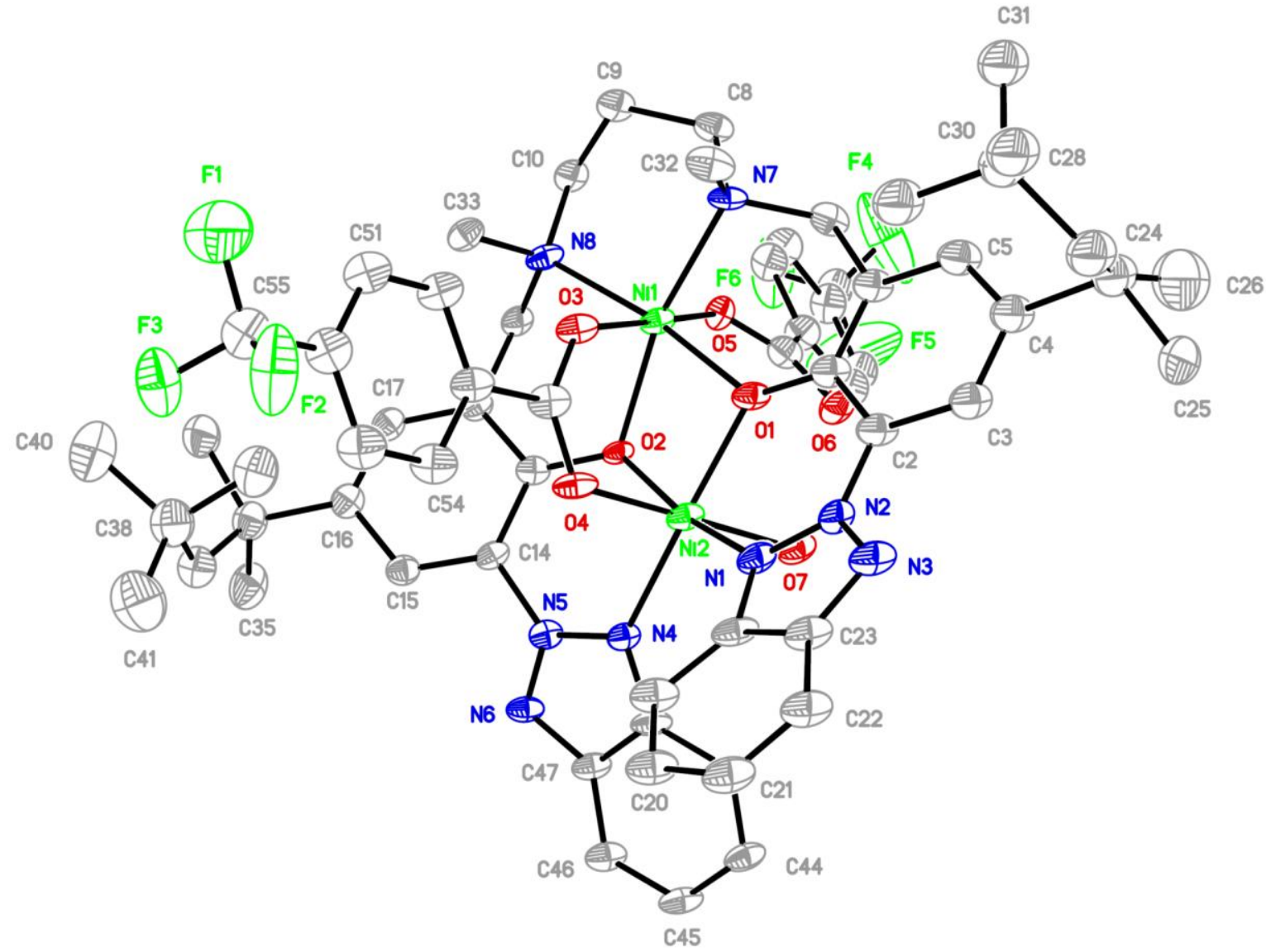

Figure S9. ORTEP drawing of complex 10 with probability ellipsoids drawn at $30 \%$ level. Hydrogen atoms are omitted for clarity. Selected bond lengths $(\AA)$ and angles (deg): $\mathrm{Ni}(1)-\mathrm{O}(1)$ 2.055(5), $\mathrm{Ni}(1)-\mathrm{O}(3)$ 2.082(5), Ni(1)-O(5) 2.088(5), Ni(1)-N(7) 2.116(5), $\mathrm{Ni}(1)-\mathrm{O}(2)$ 2.119(4), $\mathrm{Ni}(1)-\mathrm{N}(8)$ 2.145(6), Ni(2)-O(1) 1.988(5), Ni(2)-O(4) 2.025(5), $\mathrm{Ni}(2)-\mathrm{N}(4)$ 2.060(5), $\mathrm{Ni}(2)-\mathrm{O}(2)$ 2.058(4), Ni(2)-N(1) 2.077(6), Ni(2)-O(7) 2.113(6), $\mathrm{O}(1)-\mathrm{Ni}(1)-\mathrm{O}(3) \quad 82.9(2), \quad \mathrm{O}(1)-\mathrm{Ni}(1)-\mathrm{O}(5) \quad$ 102.34(19), $\quad \mathrm{O}(3)-\mathrm{Ni}(1)-\mathrm{O}(5) \quad$ 174.06(19), $\mathrm{O}(1)-\mathrm{Ni}(1)-\mathrm{N}(7)$ 89.7(2), O(3)-Ni(1)-N(7) 96.0(2), O(5)-Ni(1)-N(7) 86.8(2), O(1)-Ni(1)-O(2) 81.02(17), $\quad \mathrm{O}(3)-\mathrm{Ni}(1)-\mathrm{O}(2) \quad 91.55(18), \quad \mathrm{O}(5)-\mathrm{Ni}(1)-\mathrm{O}(2) \quad 86.60(18), \quad \mathrm{N}(7)-\mathrm{Ni}(1)-\mathrm{O}(2)$ 167.2(2), O(1)-Ni(1)-N(8) 169.2(2), O(3)-Ni(1)-N(8) 87.4(2), O(5)-Ni(1)-N(8) 87.1(2), $\mathrm{N}(7)-\mathrm{Ni}(1)-\mathrm{N}(8) \quad 96.2(2), \quad \mathrm{O}(2)-\mathrm{Ni}(1)-\mathrm{N}(8) \quad 94.48(19), \quad \mathrm{O}(1)-\mathrm{Ni}(2)-\mathrm{O}(4) \quad 96.6(2)$, $\mathrm{O}(1)-\mathrm{Ni}(2)-\mathrm{N}(4) \quad$ 168.7(2), $\quad \mathrm{O}(4)-\mathrm{Ni}(2)-\mathrm{N}(4) \quad 91.4(2), \quad \mathrm{O}(1)-\mathrm{Ni}(2)-\mathrm{O}(2) \quad 84.14(17)$, $\mathrm{O}(4)-\mathrm{Ni}(2)-\mathrm{O}(2) \quad 88.39(19), \quad \mathrm{N}(4)-\mathrm{Ni}(2)-\mathrm{O}(2) \quad 88.19(19), \quad \mathrm{O}(1)-\mathrm{Ni}(2)-\mathrm{N}(1) \quad 86.2(2)$, $\mathrm{O}(4)-\mathrm{Ni}(2)-\mathrm{N}(1) \quad 85.5(2), \quad \mathrm{N}(4)-\mathrm{Ni}(2)-\mathrm{N}(1) \quad 102.3(2), \quad \mathrm{O}(2)-\mathrm{Ni}(2)-\mathrm{N}(1) \quad 167.95(19)$, $\mathrm{O}(1)-\mathrm{Ni}(2)-\mathrm{O}(7) \quad 83.2(2), \quad \mathrm{O}(4)-\mathrm{Ni}(2)-\mathrm{O}(7) \quad 178.6(2), \quad \mathrm{N}(4)-\mathrm{Ni}(2)-\mathrm{O}(7) \quad 88.9(2)$, $\mathrm{O}(2)-\mathrm{Ni}(2)-\mathrm{O}(7)$ 93.03(19), $\mathrm{N}(1)-\mathrm{Ni}(2)-\mathrm{O}(7) 93.0(2)$. 


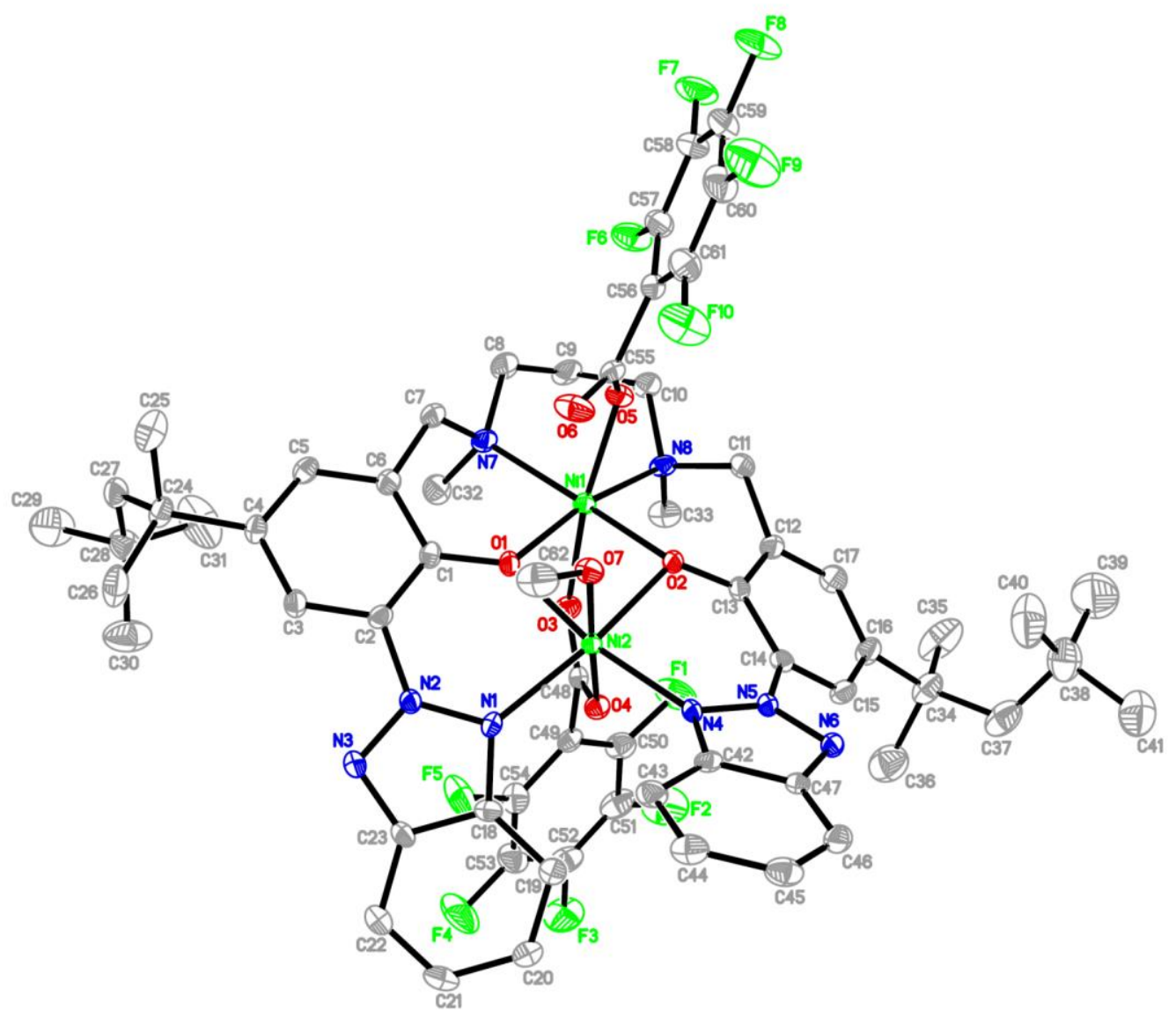

Figure S10. ORTEP drawing of complex 11 with probability ellipsoids drawn at $50 \%$ level. Hydrogen atoms are omitted for clarity. Selected bond lengths $(\AA)$ and angles (deg): $\mathrm{Ni}(1)-\mathrm{O}(1) \quad 2.037(2), \mathrm{Ni}(1)-\mathrm{O}(2)$ 2.091(2), Ni(1)-O(5) 2.124(2), Ni(1)-N(7) 2.125(3), $\mathrm{Ni}(1)-\mathrm{N}(8)$ 2.136(3), $\mathrm{Ni}(1)-\mathrm{O}(3)$ 2.156(2), Ni(2)-O(1) 2.000(2), $\mathrm{Ni}(2)-\mathrm{O}(4) \quad 2.037(2)$, $\mathrm{Ni}(2)-\mathrm{N}(4)$ 2.041(3), $\mathrm{Ni}(2)-\mathrm{O}(2)$ 2.061(2), $\mathrm{Ni}(2)-\mathrm{N}(1)$ 2.085(3), $\mathrm{Ni}(2)-\mathrm{O}(7)$ 2.093(2), $\mathrm{O}(1)-\mathrm{Ni}(1)-\mathrm{O}(2) \quad 80.84(8), \quad \mathrm{O}(1)-\mathrm{Ni}(1)-\mathrm{O}(5) \quad 104.56(9), \quad \mathrm{O}(2)-\mathrm{Ni}(1)-\mathrm{O}(5) \quad 85.48(8)$, $\mathrm{O}(1)-\mathrm{Ni}(1)-\mathrm{N}(7) \quad 88.08(9), \quad \mathrm{O}(2)-\mathrm{Ni}(1)-\mathrm{N}(7) \quad 164.90(10), \quad \mathrm{O}(5)-\mathrm{Ni}(1)-\mathrm{N}(7) \quad 87.43(10)$, $\mathrm{O}(1)-\mathrm{Ni}(1)-\mathrm{N}(8) \quad 168.97(10), \quad \mathrm{O}(2)-\mathrm{Ni}(1)-\mathrm{N}(8) \quad 95.70(9), \quad \mathrm{O}(5)-\mathrm{Ni}(1)-\mathrm{N}(8) \quad 85.51(10)$, $\mathrm{N}(7)-\mathrm{Ni}(1)-\mathrm{N}(8) \quad 97.01(10), \quad \mathrm{O}(1)-\mathrm{Ni}(1)-\mathrm{O}(3) \quad 82.34(9), \quad \mathrm{O}(2)-\mathrm{Ni}(1)-\mathrm{O}(3) \quad 91.10(8)$, $\mathrm{O}(5)-\mathrm{Ni}(1)-\mathrm{O}(3) \quad$ 171.66(9), $\quad \mathrm{N}(7)-\mathrm{Ni}(1)-\mathrm{O}(3) \quad 97.60(10), \quad \mathrm{N}(8)-\mathrm{Ni}(1)-\mathrm{O}(3) \quad 87.28(9)$, $\mathrm{O}(1)-\mathrm{Ni}(2)-\mathrm{O}(4) \quad 93.97(9), \quad \mathrm{O}(1)-\mathrm{Ni}(2)-\mathrm{N}(4) \quad 170.21(9), \quad \mathrm{O}(4)-\mathrm{Ni}(2)-\mathrm{N}(4) \quad 90.34(10)$, $\mathrm{O}(1)-\mathrm{Ni}(2)-\mathrm{O}(2) \quad 82.46(8), \quad \mathrm{O}(4)-\mathrm{Ni}(2)-\mathrm{O}(2) \quad 87.83(9), \quad \mathrm{N}(4)-\mathrm{Ni}(2)-\mathrm{O}(2) \quad 88.93(9)$, $\mathrm{O}(1)-\mathrm{Ni}(2)-\mathrm{N}(1) \quad 87.60(9), \quad \mathrm{O}(4)-\mathrm{Ni}(2)-\mathrm{N}(1) \quad 83.43(10), \quad \mathrm{N}(4)-\mathrm{Ni}(2)-\mathrm{N}(1) \quad$ 101.64(10), $\mathrm{O}(2)-\mathrm{Ni}(2)-\mathrm{N}(1) \quad 166.28(10), \quad \mathrm{O}(1)-\mathrm{Ni}(2)-\mathrm{O}(7) \quad 86.02(9), \quad \mathrm{O}(4)-\mathrm{Ni}(2)-\mathrm{O}(7) \quad$ 177.91(9), $\mathrm{N}(4)-\mathrm{Ni}(2)-\mathrm{O}(7)$ 89.35(10), O(2)-Ni(2)-O(7) 90.10(9), N(1)-Ni(2)-O(7) 98.66(10). 


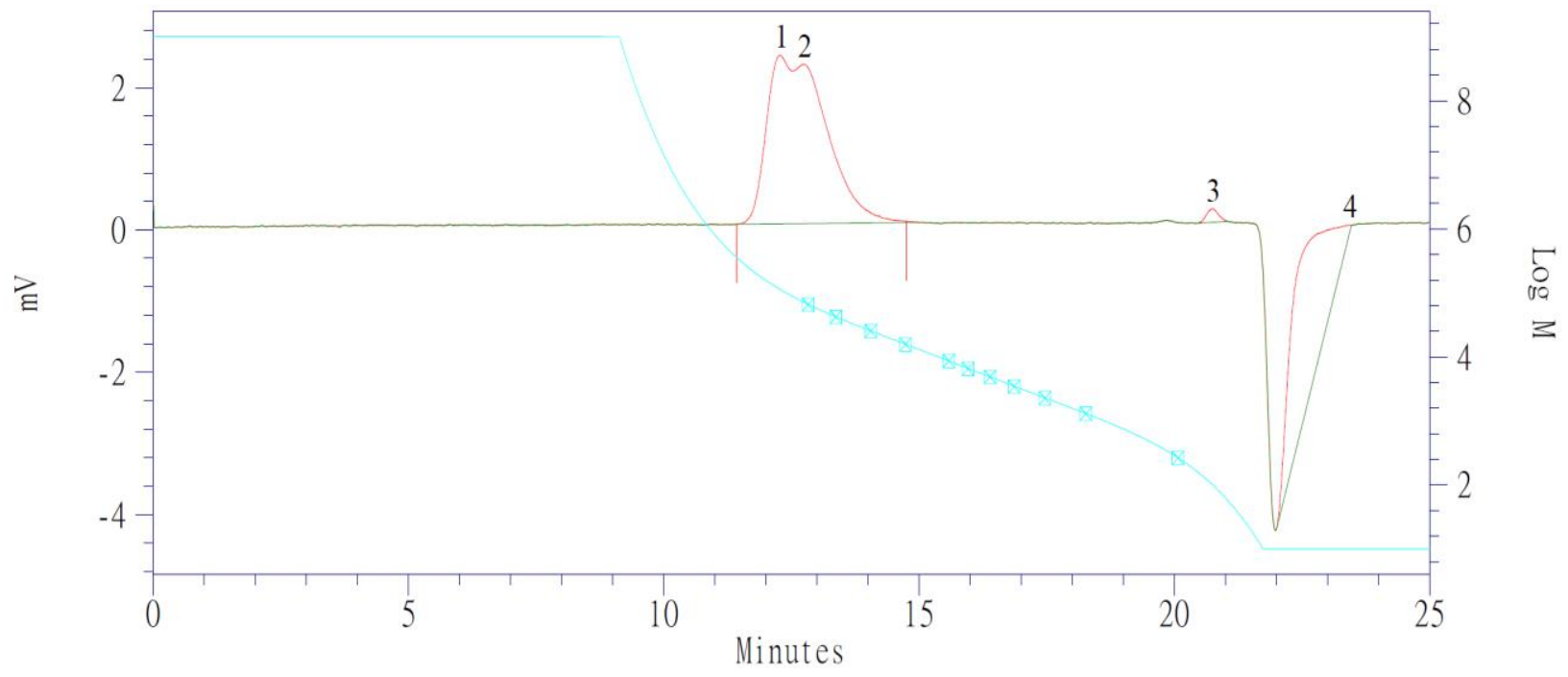

Figure S11. GPC traces for the afforded PCHC with a bimodal molecular weight distribution catalyzed by dinickel complex 6 (Table 2, entry 4). 


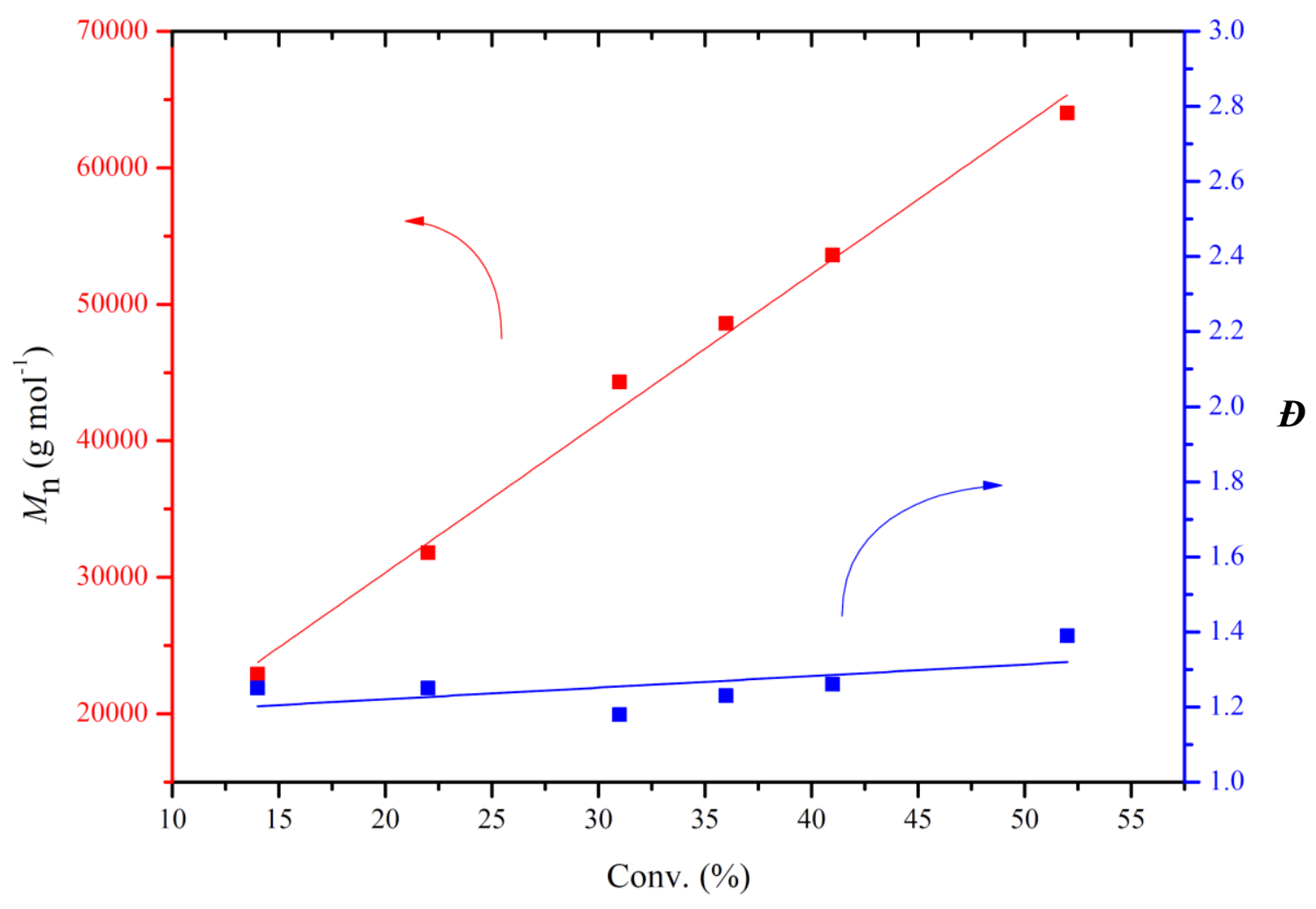

Figure S12. The plot of $M_{\mathrm{n}}(\square)$ and $D(\square)$ (determined from GPC analysis) versus CHO conversion for the $\mathrm{CO}_{2}$-copolymerization of $\mathrm{CHO}$ using dinickel complex $\mathbf{6}$ as the catalyst $\left([\mathrm{CHO}]_{0} /[6]_{0}=5000\right)$ at $80{ }^{\circ} \mathrm{C}$ and 20.7 bar $\mathrm{CO}_{2}$. 

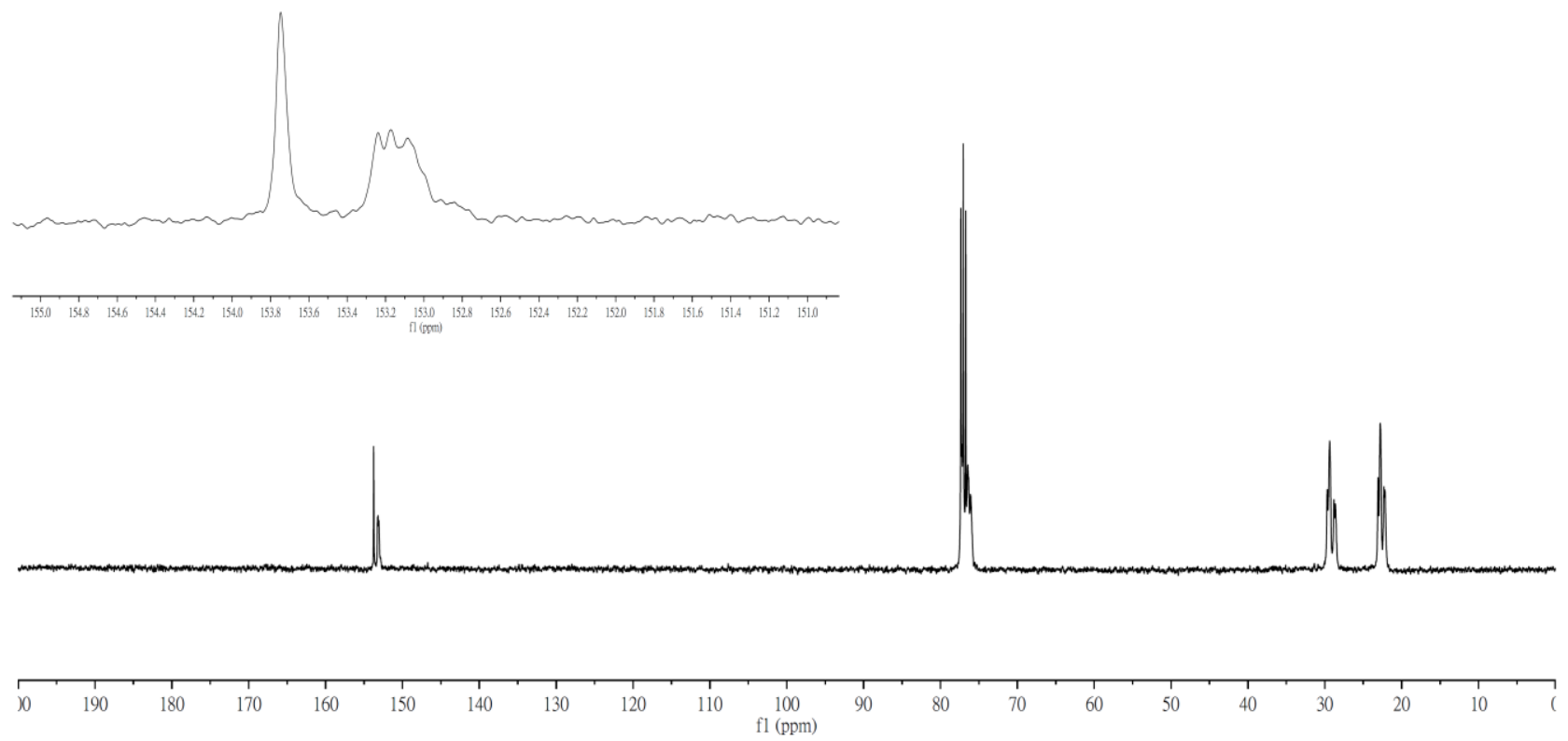

Figure S13. Carbonyl region of ${ }^{13} \mathrm{C}$ NMR spectra of the PCHC copolymer (Table 2, entry 4) in $\mathrm{CDCl}_{3}$. (inset) Expanded carbonyl region. 


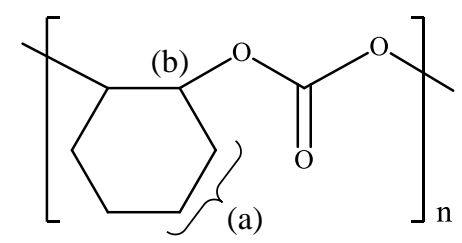

(a)

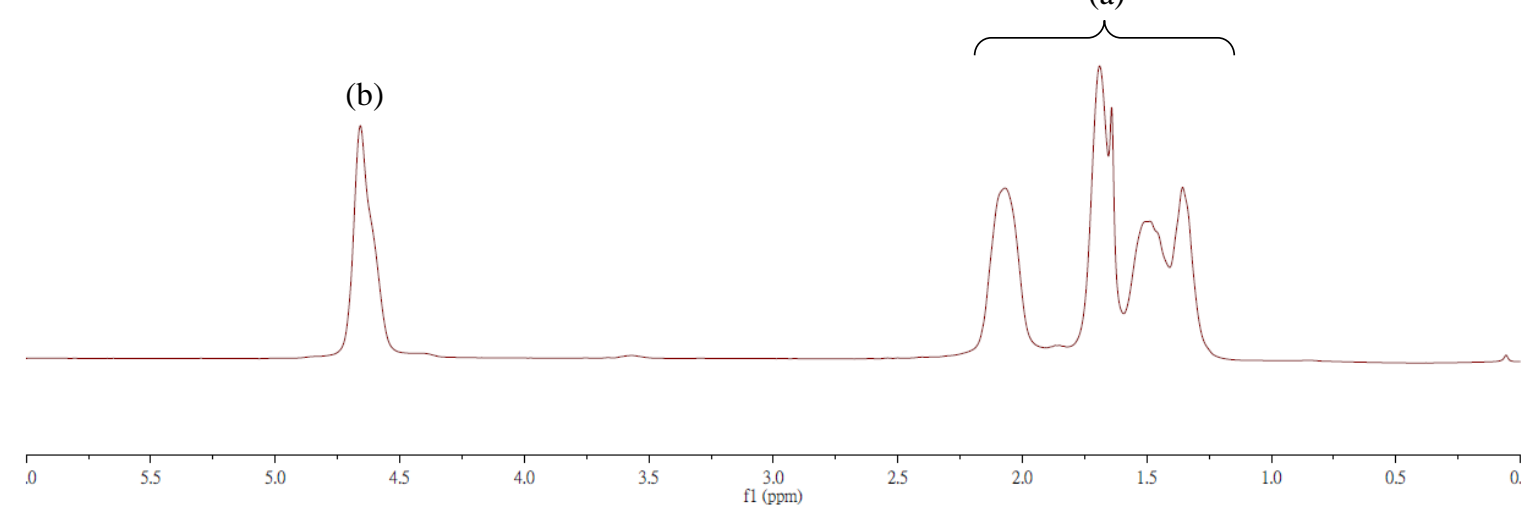

Figure S14. ${ }^{1} \mathrm{H}$ NMR spectrum of the purified copolymer produced by utilizing dinickel complex 6 (Table 3, entry 2) in $\mathrm{CDCl}_{3}$. The peak at $\delta 4.65 \mathrm{ppm}$ is assigned to the methine protons in $\mathrm{PCHC}$, and no significant signal at 3.3-3.5 ppm suggests $>99 \%$ carbonate carbonates in $\mathrm{PCHC}$. 


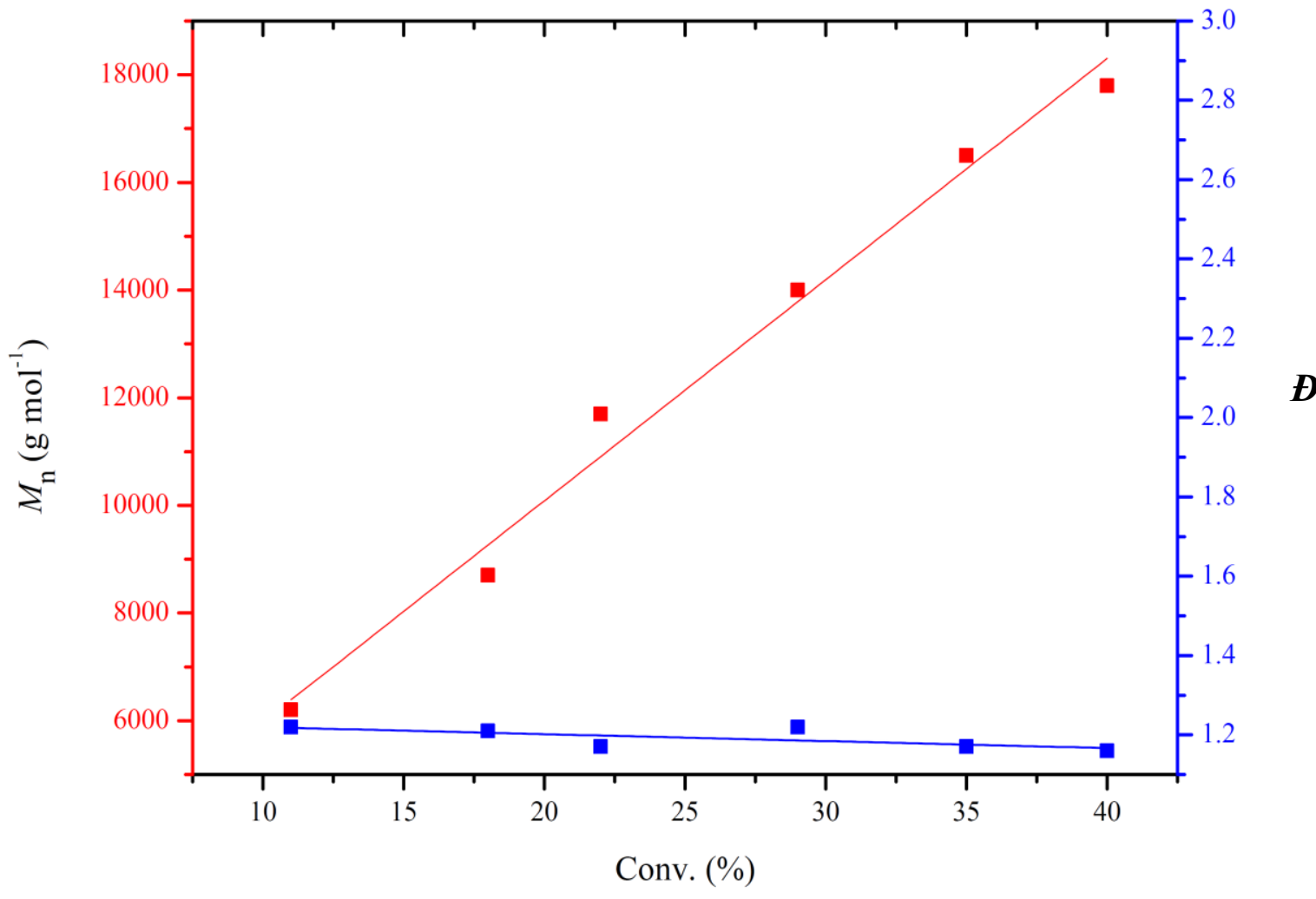

Figure S15. The plot of $M_{\mathrm{n}}(\square)$ and $Ð(\square)$ (determined from GPC analysis) versus $\mathrm{CHO}$ conversion for the $\mathrm{CO}_{2}$-copolymerization of $\mathrm{CHO}$ employing dinickel complex $\mathbf{6}$ as the catalyst $\left([\mathrm{CHO}]_{0} /[6]_{0}=1600\right)$ at $100{ }^{\circ} \mathrm{C}$ and 1 atm of $\mathrm{CO}_{2}$ pressure. 


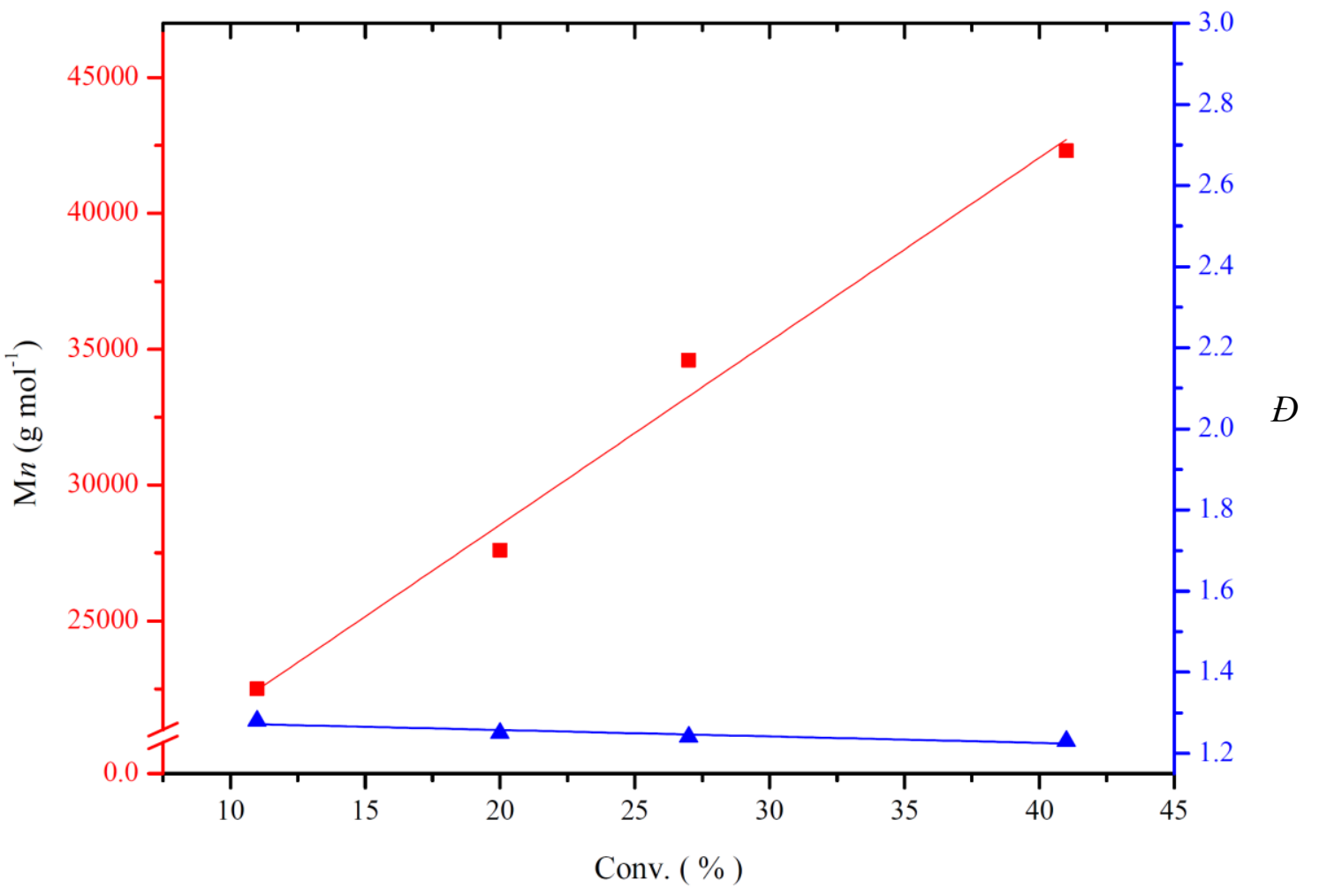

Figure S16. The plot of $M_{\mathrm{n}}(\boldsymbol{\square})$ and PDI ( $\left.\boldsymbol{\Delta}\right)$ (determined from GPC analysis) versus VCHO conversion for the $\mathrm{CO}_{2}$-copolymerization of $\mathrm{VCHO}$ using dinickel complex $\mathbf{6}$ as the catalyst $\left([\mathrm{CHO}]_{0} /[6]_{0}=5000\right)$ at $80{ }^{\circ} \mathrm{C}$ and 20.7 bar $\mathrm{CO}_{2}$. 


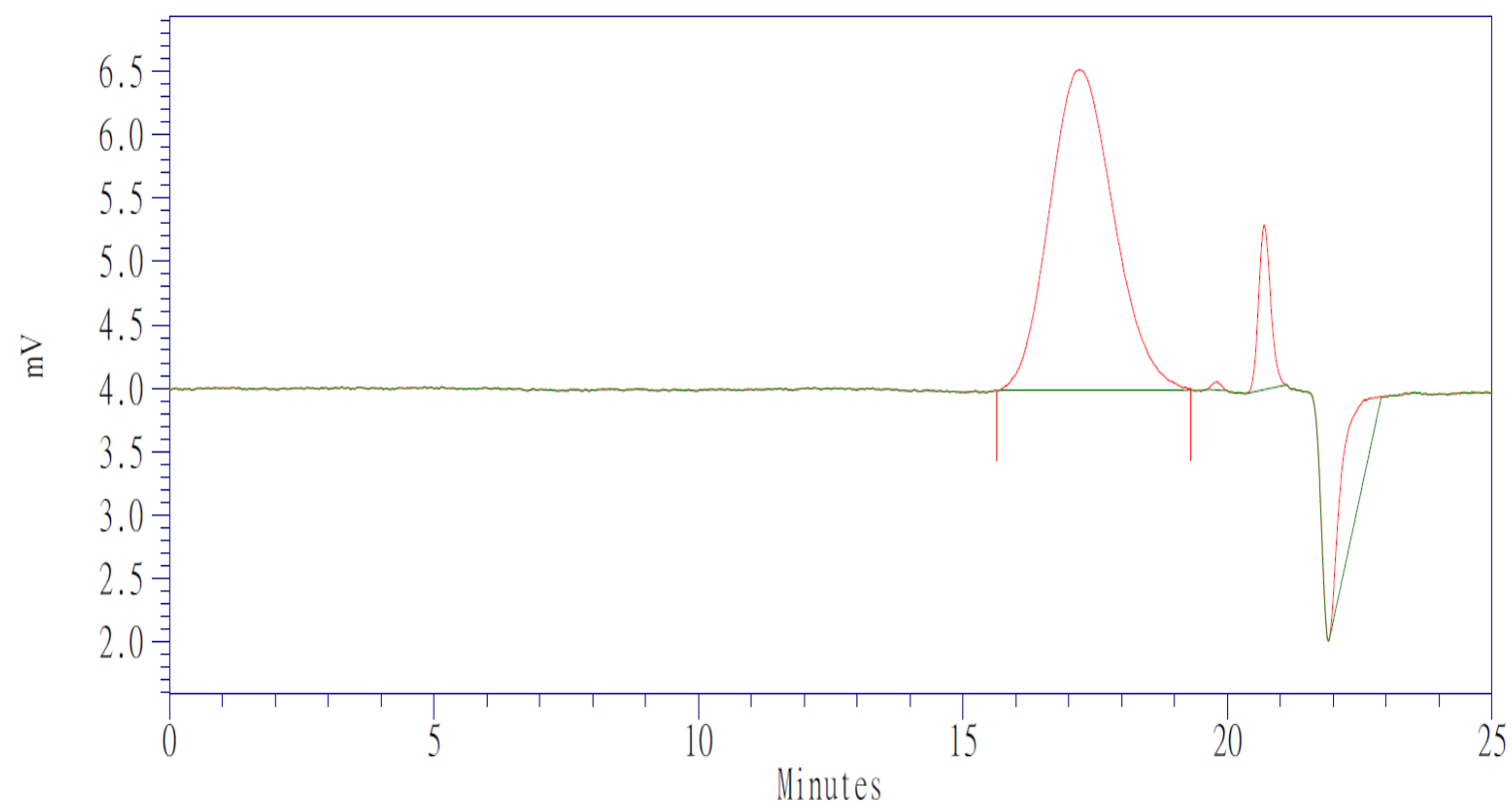

Figure S17. GPC traces for the produced PCHC with a unimodal molecular weight distribution catalyzed by dinickel complex 6 (Table 4, entry 8 ). 
(a)

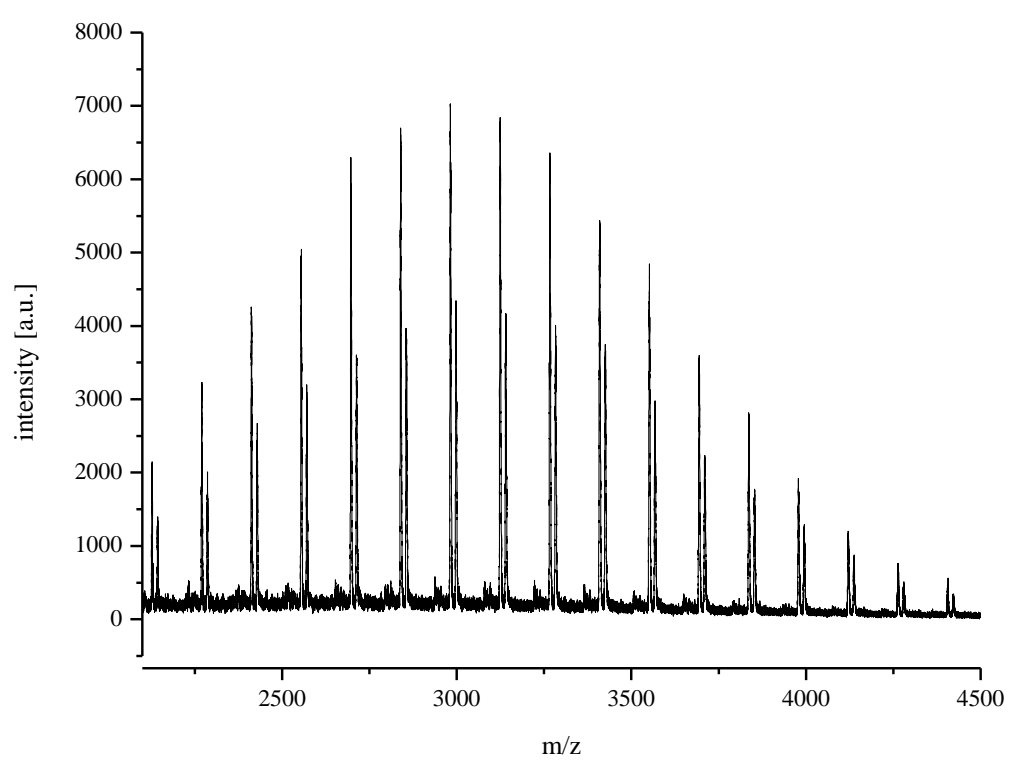

(b)<smiles>[Y]C1CCCC(OC(=O)OC2CCCC2O)C1C1OC1O</smiles><smiles>O=C(OC1CCCC1)C1CC2CCCC2C1O</smiles>

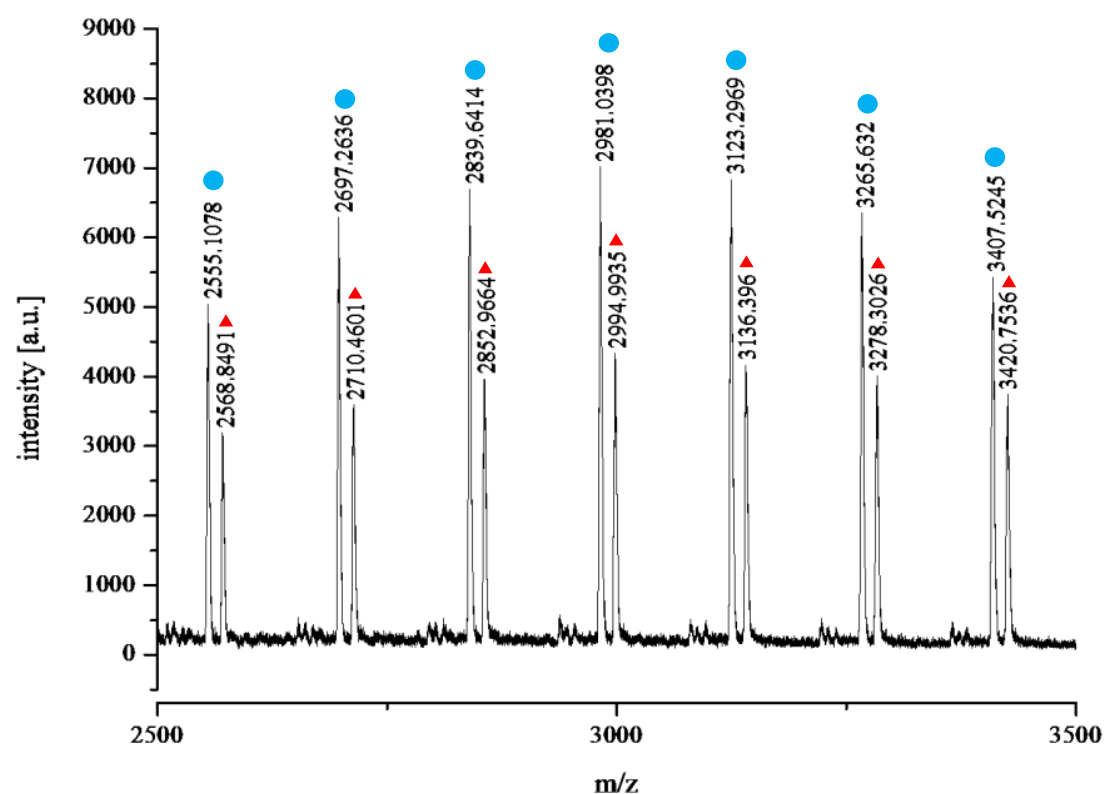

Figure S18. The MALDI-TOF spectrum of the obtained PCHC catalyzed by dinickel complex 6 with the addition of water as the chain transfer agent (Table 4, entry 8). 
(a)

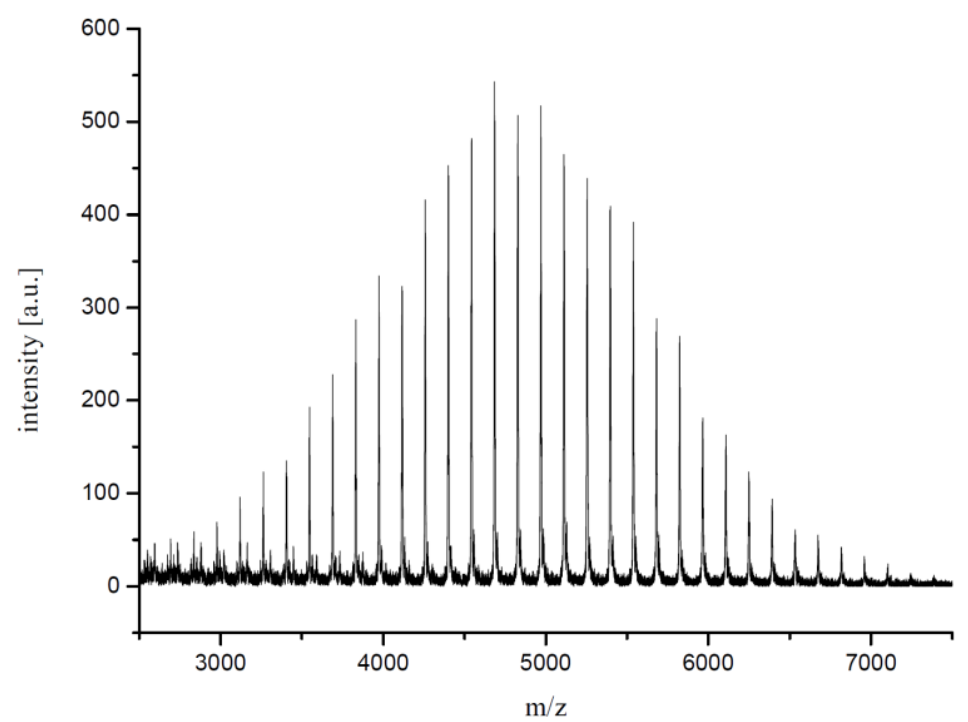

(b)

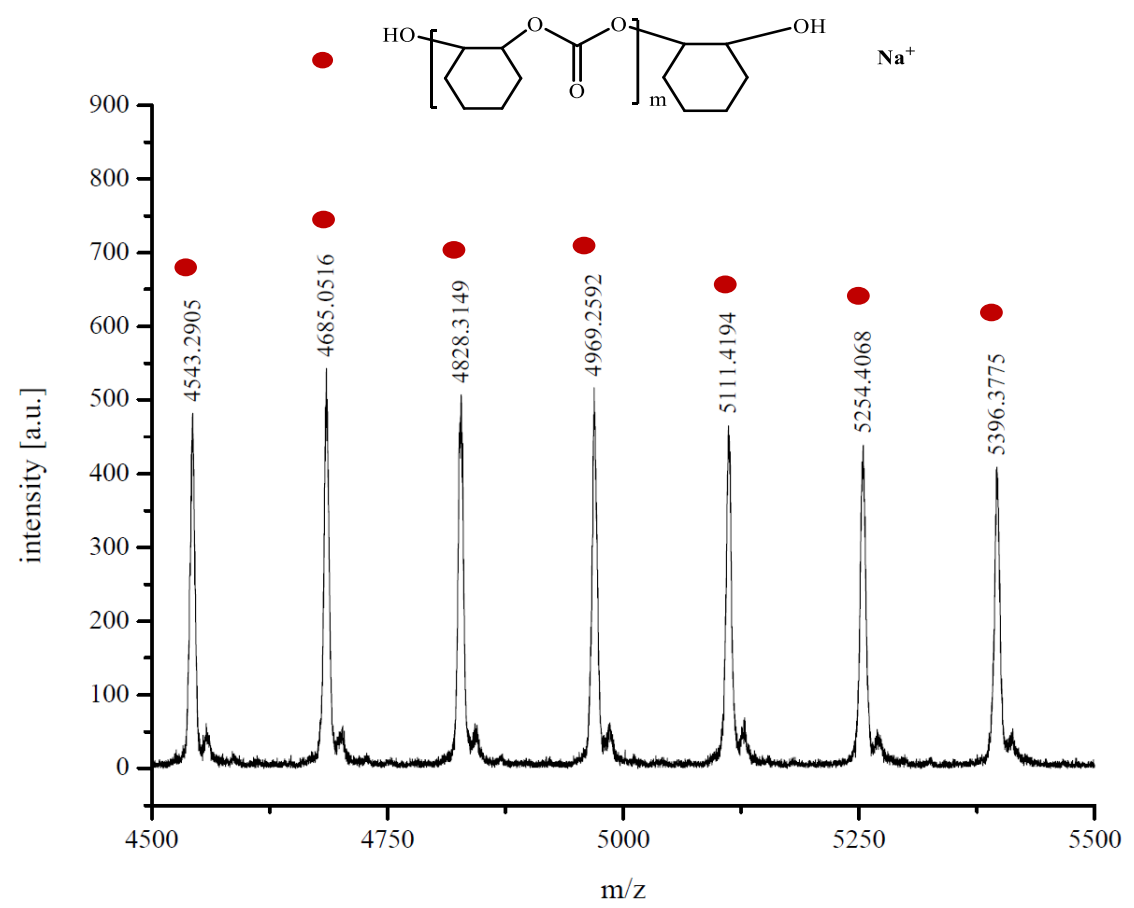

Figure S19. The MALDI-TOF spectrum of the afforded PCHC polyol catalyzed by dinickel complex 6 (Table 4, entry 9). 
(a)

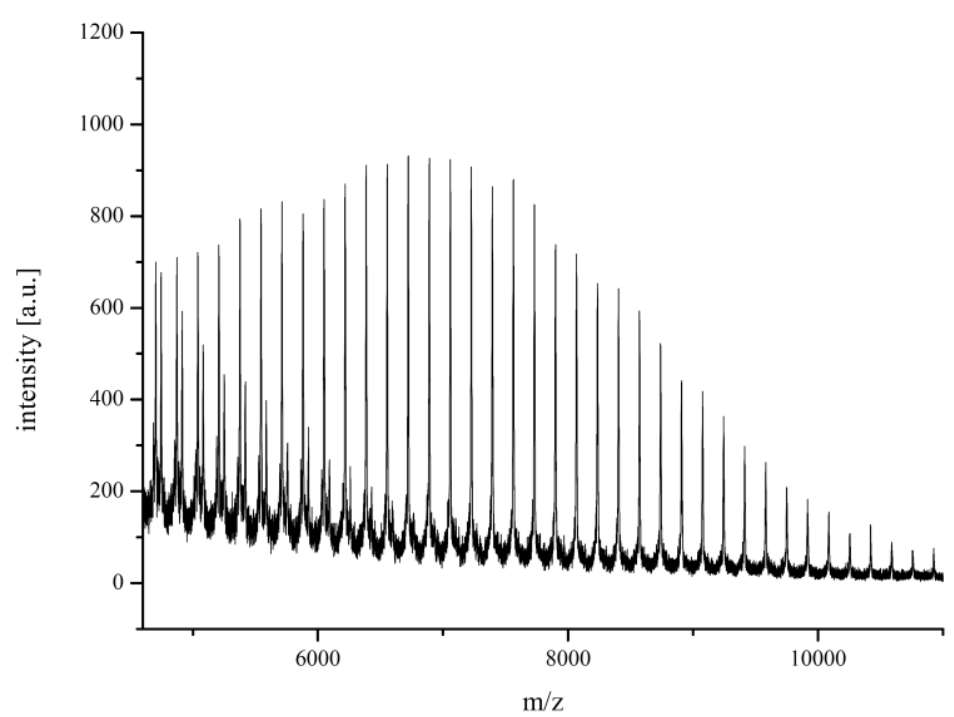

(b)

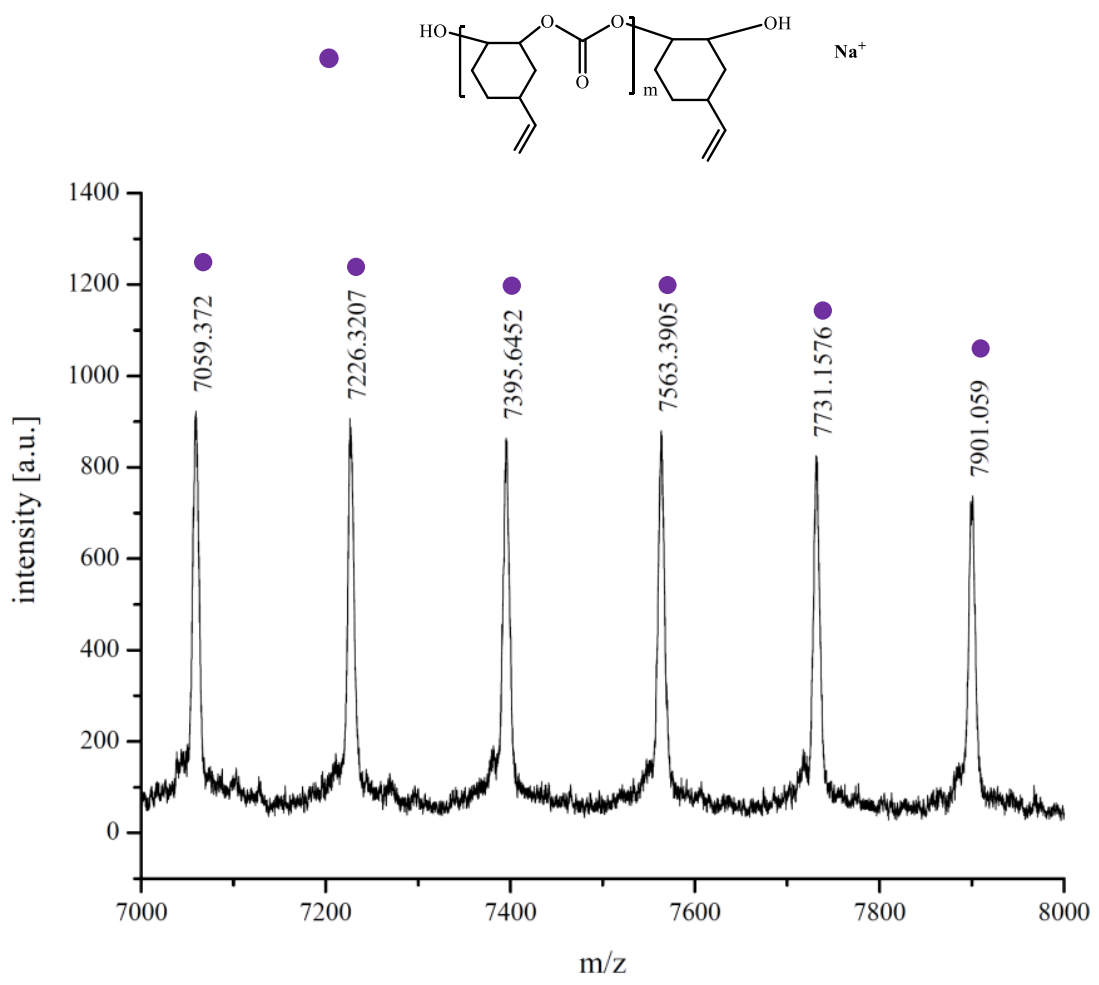

Figure S20. The MALDI-TOF spectrum of the yielded PVCHC polyol catalyzed by dinickel complex 6 (Table 4, entry 10). 
(a)

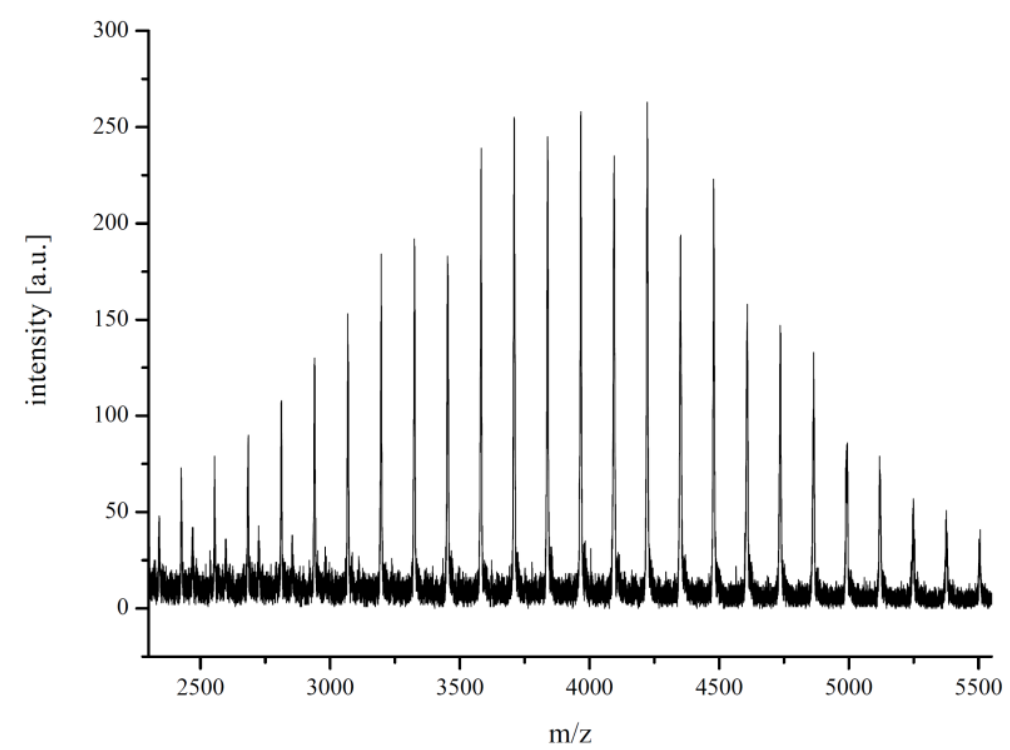

(b)

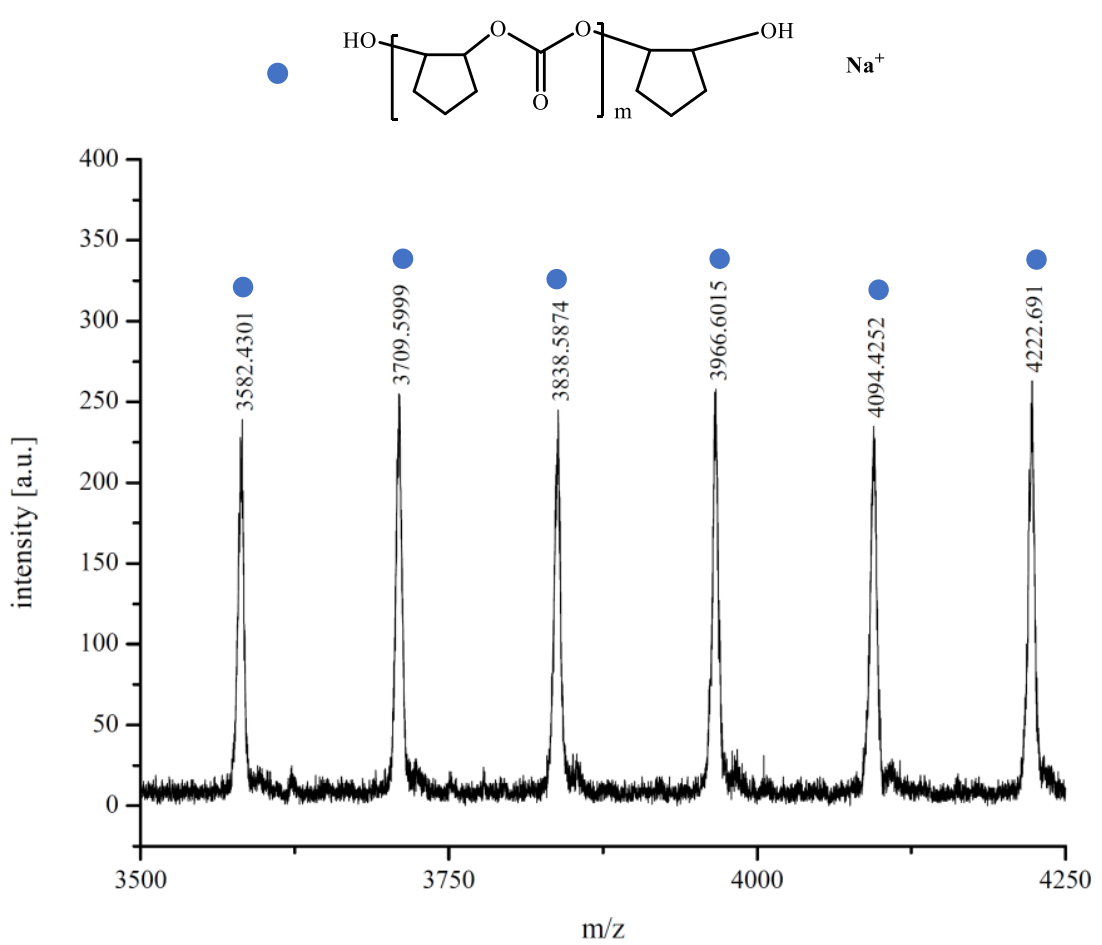

Figure S21. The MALDI-TOF spectrum of the produced PCPC polyol catalyzed by dinickel complex 6 (Table 4, entry 11). 


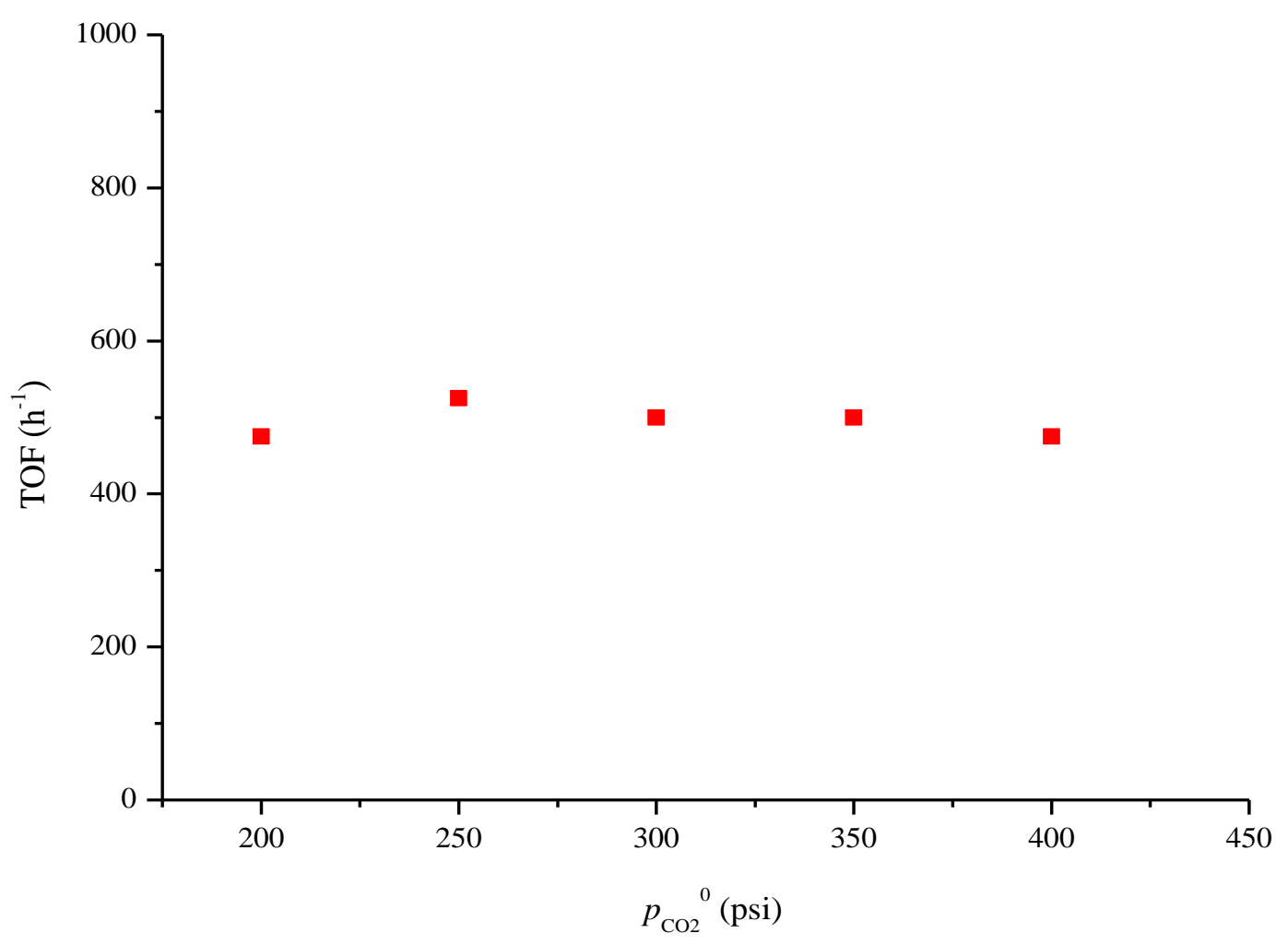

Figure S22. Plots of TOFs with different initial $\mathrm{CO}_{2}$ pressures utilizing dinickel complex 6 $(0.02 \mathrm{~mol} \%)$ at $80^{\circ} \mathrm{C}$ for $4 \mathrm{~h}$. 
- $\quad 0.0200 \mathrm{~mole} \%$

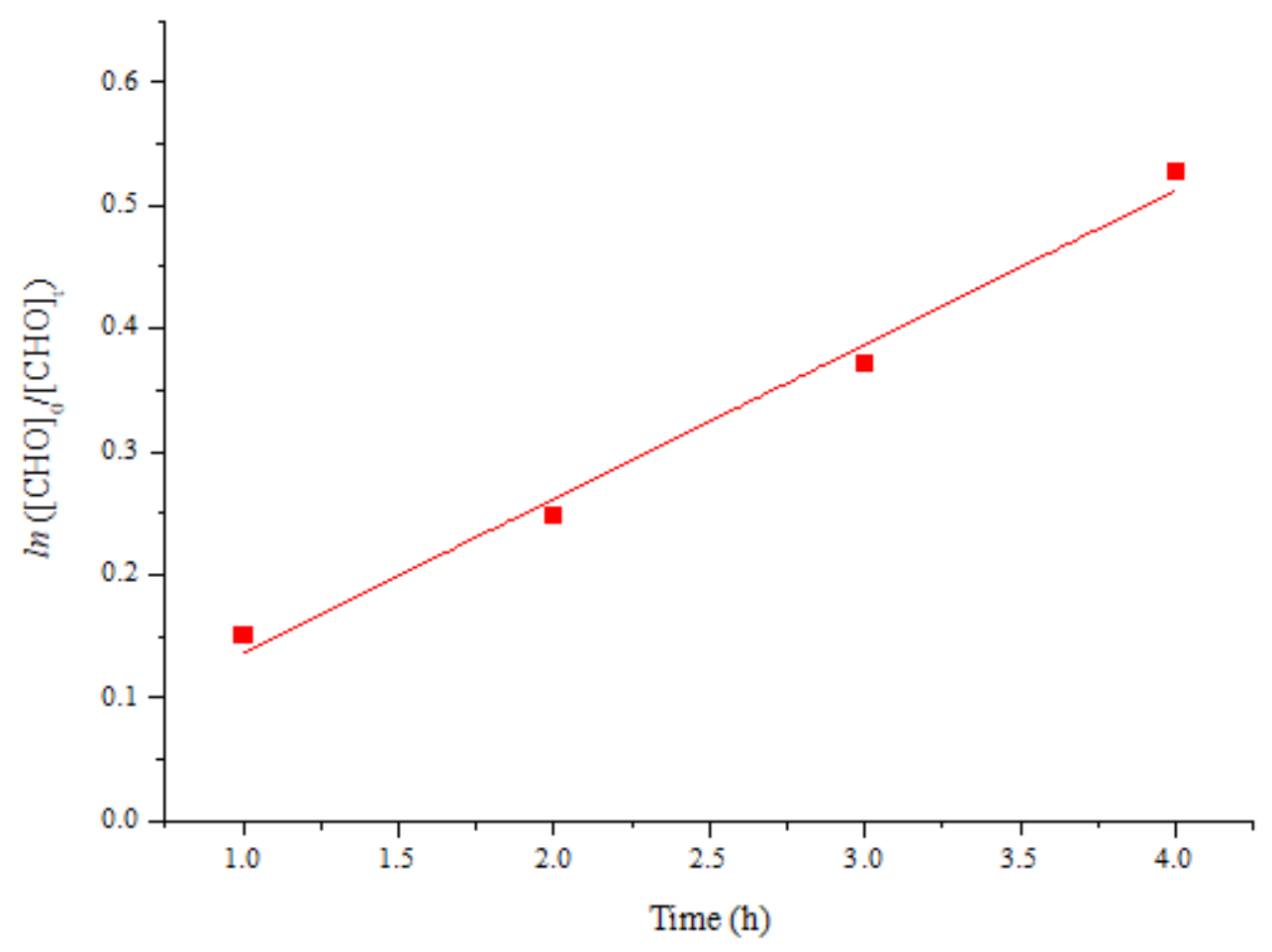

Figure S23. The kinetic plot of $\ln \left([\mathrm{CHO}]_{0} /[\mathrm{CHO}]_{t}\right)$ versus time for the copolymerization of $\mathrm{CHO}$ and $\mathrm{CO}_{2}$ by dinickel complex 6 at $80{ }^{\circ} \mathrm{C}$ and an initial $\mathrm{CO}_{2}$ pressure of 20.7 bar using a catalyst loading of $0.02 \mathrm{~mol} \%$. 


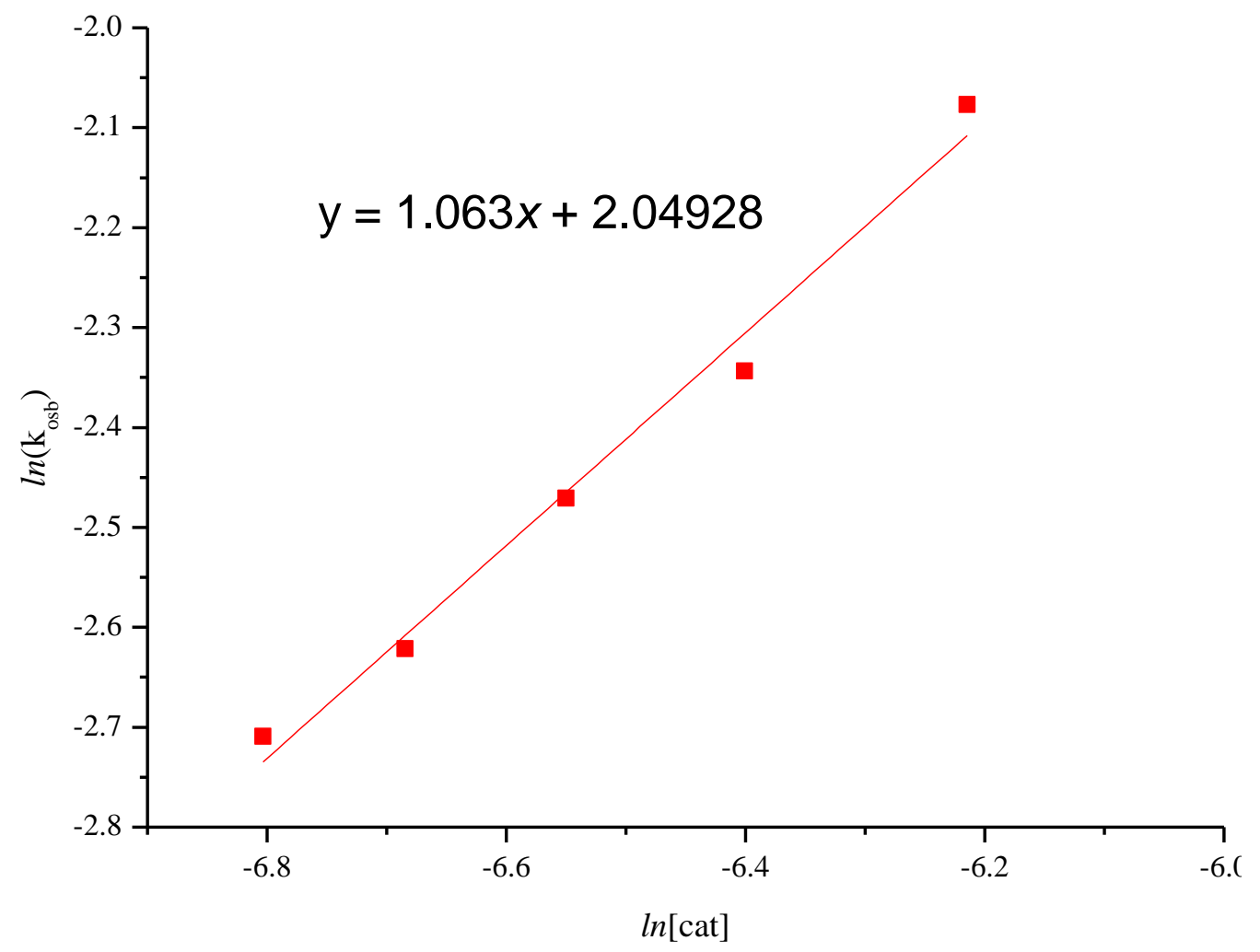

Figure S24. A linear plot of $\ln k_{\mathrm{obs}}$ versus $\ln [6]$ displaying the first order dependence on dinickel catalyst concentration. All polymerizations were performed at $80{ }^{\circ} \mathrm{C}$ and an initial $\mathrm{CO}_{2}$ pressure of 20.7 bar. 


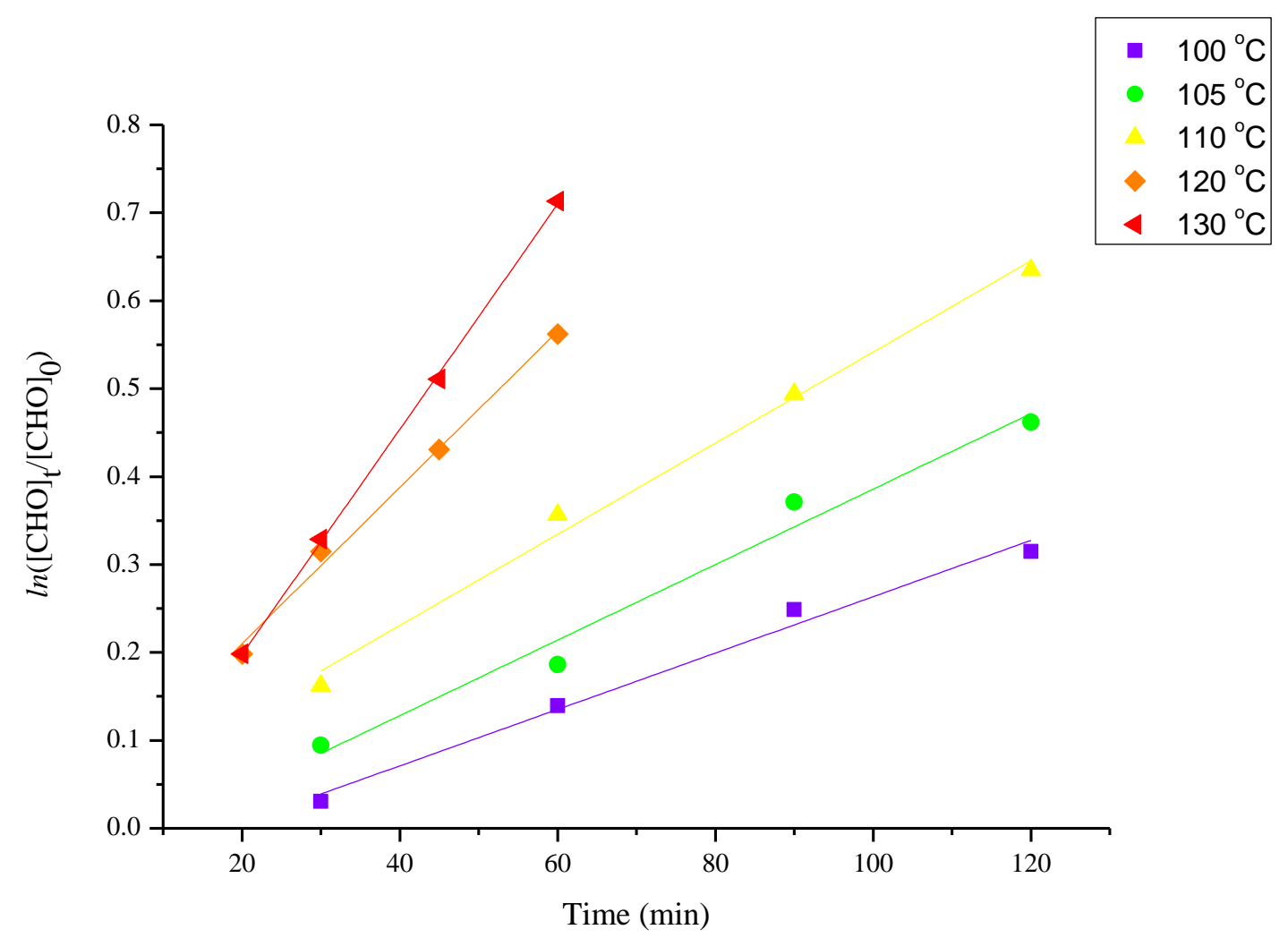

Figure S25. Kinetic plots of $\ln \left([\mathrm{CHO}]_{0} /[\mathrm{CHO}]_{t}\right)$ versus time for $\mathrm{CO}_{2}$-copolymerization of CHO by dinickel complex 6 at an initial $\mathrm{CO}_{2}$ pressure of 20.7 bar and a catalyst loading of 0.01 mol\% with various temperatures $\left(100,105,110,120\right.$ and $\left.130{ }^{\circ} \mathrm{C}\right)$. 
Table S1. Selected Bond Lengths and Angles of Nickel Complexes 1-11 and $\mathrm{A}^{35}$

\begin{tabular}{|c|c|c|c|c|c|c|}
\hline & 1 & 2 & 3 & 4 & 5 & 6 \\
\hline $\mathrm{Ni}-\mathrm{O}($ phenoxy) & $2.061(2)$ & $2.033(5)$ & $2.055(3)$ & $2.043(5)$ & $2.042(4)$ & $2.050(7)$ \\
\hline Ni-O(carboxylate) & $2.067(2)$ & $2.079(5)$ & $2.076(3)$ & $2.083(5)$ & $2.091(4)$ & $2.057(9)$ \\
\hline Ni-N(amine) & $2.156(3)$ & $2.112(6)$ & $2.128(3)$ & $2.128(6)$ & $2.122(5)$ & $2.140(9)$ \\
\hline Ni-N(benzotriazole) & $2.077(3)$ & $2.037(6)$ & $2.080(3)$ & $2.053(6)$ & $2.052(4)$ & $2.064(9)$ \\
\hline $\mathrm{O}(1)-\mathrm{Ni}(1)-\mathrm{N}(8)$ & $169.79(9)$ & $165.4(2)$ & $172.63(12)$ & $169.0(2)$ & $168.82(16)$ & $169.8(3)$ \\
\hline $\mathrm{O}(2)-\mathrm{Ni}(1)-\mathrm{N}(7)$ & $166.67(8)$ & $169.0(2)$ & $169.67(12)$ & $165.5(2)$ & $165.08(16)$ & $166.1(3)$ \\
\hline $\mathrm{O}(3)-\mathrm{Ni}(1)-\mathrm{O}(5)$ & $172.80(9)$ & $176.8(2)$ & $177.26(12)$ & $174.33(19)$ & 175.11(15) & $173.3(3)$ \\
\hline $\mathrm{O}(3)-\mathrm{Ni}(1)-\mathrm{O}(7)$ & - & - & - & - & - & - \\
\hline $\mathrm{O}(1)-\mathrm{Ni}(2)-\mathrm{N}(4)$ & $166.02(9)$ & $167.1(2)$ & $164.63(13)$ & $167.1(2)$ & $166.40(17)$ & $168.9(3)$ \\
\hline $\mathrm{O}(2)-\mathrm{Ni}(2)-\mathrm{N}(1)$ & $170.29(8)$ & $169.9(2)$ & $169.65(12)$ & $165.5(2)$ & $165.88(16)$ & $167.2(3)$ \\
\hline $\mathrm{O}(4)-\mathrm{Ni}(2)-\mathrm{O}(7)$ & $178.24(8)$ & $175.2(2)$ & 177.11(11) & $177.57(19)$ & $177.03(15)$ & $178.2(3)$ \\
\hline $\mathrm{O}(4)-\mathrm{Ni}(2)-\mathrm{O}(5)$ & - & - & - & - & - & - \\
\hline $\mathrm{Ni}(1) \ldots \mathrm{Ni}(2)$ & 3.021 & 3.006 & 3.035 & 3.043 & 3.039 & 3.013 \\
\hline
\end{tabular}

\begin{tabular}{lllllll}
\hline & $\mathbf{7}$ & $\mathbf{8}$ & $\mathbf{9}$ & $\mathbf{1 0}$ & $\mathbf{1 1}$ & $\mathbf{A}$ \\
\hline $\mathrm{Ni}-\mathrm{O}($ phenoxy) & $2.0454(19)$ & $2.050(10)$ & $2.046(2)$ & $2.055(5)$ & $2.047(2)$ & $2.041(3)$ \\
$\mathrm{Ni}-\mathrm{O}($ carboxylate) & $2.0586(19)$ & $2.065(10)$ & $2.062(2)$ & $2.065(5)$ & $2.106(2)$ & $2.070(3)$ \\
$\mathrm{Ni}-\mathrm{N}($ amine) & $2.135(2)$ & $2.122(12)$ & $2.129(3)$ & $2.131(6)$ & $2.131(3)$ & $2.127(4)$ \\
$\mathrm{Ni}-\mathrm{N}($ benzotriazole) & $2.048(2)$ & $2.067(13)$ & $2.054(3)$ & $2.069(6)$ & $2.063(3)$ & $2.065(4)$ \\
& & & & & & \\
$\mathrm{O}(1)-\mathrm{Ni}(1)-\mathrm{N}(8)$ & $169.91(8)$ & $172.0(4)$ & $167.33(9)$ & $169.2(2)$ & $168.97(10)$ & $169.82(13)$ \\
$\mathrm{O}(2)-\mathrm{Ni}(1)-\mathrm{N}(7)$ & $167.70(8)$ & $167.2(4)$ & $168.43(10)$ & $167.2(2)$ & $164.9(1)$ & $164.46(12)$ \\
$\mathrm{O}(3)-\mathrm{Ni}(1)-\mathrm{O}(5)$ & $174.61(8)$ & - & $174.68(9)$ & $174.06(19)$ & $171.66(9)$ & - \\
$\mathrm{O}(3)-\mathrm{Ni}(1)-\mathrm{O}(7)$ & - & $170.4(4)$ & - & - & & $174.95(13)$ \\
$\mathrm{O}(1)-\mathrm{Ni}(2)-\mathrm{N}(4)$ & $169.83(9)$ & $169.8(4)$ & $167.95(9)$ & $168.7(2)$ & $170.21(9)$ & $169.57(13)$ \\
$\mathrm{O}(2)-\mathrm{Ni}(2)-\mathrm{N}(1)$ & $167.87(9)$ & $167.2(5)$ & $169.98(10)$ & $167.95(19)$ & $166.28(10)$ & $166.66(13)$ \\
$\mathrm{O}(4)-\mathrm{Ni}(2)-\mathrm{O}(7)$ & $177.87(8)$ & - & $175.45(8)$ & $178.6(2)$ & $177.91(9)$ & - \\
$\mathrm{O}(4)-\mathrm{Ni}(2)-\mathrm{O}(5)$ & - & $171.1(4)$ & - & - & - & $171.14(12)$ \\
& & & & & & 3.001 \\
$\mathrm{Ni}(1) \ldots \mathrm{Ni}(2)$ & 2.9995 & 2.993 & 3.014 & 3.027 & 3.052 &
\end{tabular}


Table S2. Coupling of $\mathrm{CO}_{2}$ and Terminal Epoxides Catalyzed by Dinickel Complex 6<smiles>[R]C1COC(=O)O1</smiles>

$$
\begin{array}{ll}
\mathrm{R}=\text { methyl, } \mathrm{PO} & \mathrm{R}=\text { methyl } \\
\mathrm{R}=\text { phenyl, } \mathrm{SO} & \mathrm{R}=\text { phenyl } \\
\mathrm{R}=\mathrm{n} \text {-butyl, } \mathrm{HO} & \mathrm{R}=\mathrm{n} \text {-butyl } \\
\mathrm{R}=\mathrm{CH}_{2} \mathrm{O}^{\mathrm{t}} \text { butyl, }{ }^{\mathrm{t}} \mathrm{BGE} & \mathrm{R}=\mathrm{CH}_{2} \mathrm{O}^{\mathrm{t}} \text { butyl }
\end{array}
$$

\begin{tabular}{cccccc}
\hline Entry & Epoxide substrate & $\begin{array}{c}\text { \% epoxide } \\
\text { Conversion }^{a}\end{array}$ & $\begin{array}{c}\% \text { Cyclic carbonate } \\
\text { selectivity }\end{array}$ & TON $^{b}$ & TOF $^{c}$ \\
\hline S1 & PO & 60 & $>99$ & 3000 & 500 \\
S2 & SO & 57 & $>99$ & 2850 & 475 \\
S3 & HO & 26 & $>99$ & 1300 & 217 \\
S4 & tBGE & 36 & $>99$ & 1800 & 300 \\
\hline
\end{tabular}

Coupling conditions: $0.02 \mathrm{~mol} \%$ catalyst, 20.7 bar initial $\mathrm{CO}_{2}, 140{ }^{\circ} \mathrm{C}, 6 \mathrm{~h}$. Epoxide substrates: propylene oxide (PO), styrene oxide (SO), 1-hexene oxide (HO), tert-butyl glycidyl ether ('BGE). ${ }^{a}$ Determined by ${ }^{1} \mathrm{H}$ NMR spectroscopy. ${ }^{b} \mathrm{TON}=$ number of moles of epoxide consumed per mole of catalyst. ${ }^{c} \mathrm{TOF}=\mathrm{TON}$ per hour. 
Table S3. Copolymerization of $\mathrm{CO}_{2}$ and CHO Mediated by Dinickel Complex 6 Under Different Initial $\mathrm{CO}_{2}$ Pressures for $4 \mathrm{~h}$

\begin{tabular}{cccccc}
\hline Entry & $\begin{array}{r}\text { Pressure } \\
\text { psi }\end{array}$ & $\begin{array}{r}\% \text { CHO } \\
\text { Conv. }^{a}\end{array}$ & $\begin{array}{r}\% \text { Copolymer }^{a} \\
\left(\% \text { carbonate }^{b}\right.\end{array}$ & TON $^{c}$ & $\begin{array}{r}\text { TOF } \\
/ \mathrm{h}^{-1 d}\end{array}$ \\
\hline S1 & 200 & 38 & $>99(>99)$ & 1900 & 475 \\
S2 & 250 & 42 & $>99(>99)$ & 2100 & 525 \\
S3 & 300 & 41 & $>99(>99)$ & 2050 & 513 \\
S4 & 350 & 41 & $>99(>99)$ & 2050 & 513 \\
S5 & 400 & 38 & $>99(>99)$ & 1900 & 475 \\
\hline
\end{tabular}

Copolymerization conditions: $0.02 \mathrm{~mol} \%$ catalyst, $156.25 \mathrm{mmol} \mathrm{CHO}, \mathrm{T}=80{ }^{\circ} \mathrm{C} .{ }^{a}$ Based on ${ }^{1} \mathrm{H}$ NMR analysis of the reaction mixture. ${ }^{b}$ Based on ${ }^{1} \mathrm{H}$ NMR determination of the purified copolymers. ${ }^{c} \mathrm{TON}=$ number of moles of $\mathrm{CHO}$ consumed per mole of catalyst. ${ }^{d} \mathrm{TOF}=\mathrm{TON}$ per hour. 
Table S4. Kinetic Studies of $\mathrm{CO}_{2} / \mathrm{CHO}$ Copolymerization Mediated by Dinickel Complex 6 at Different Monomer-to-Catalyst Ratio (5000, 6000, 7000, 8000 and 9000) $)^{a}$

\begin{tabular}{|c|c|c|c|c|}
\hline Entry & {$[\mathrm{CHO}]_{0}:[\mathbf{6}]_{0}$} & Time/h & $\%$ CHO Conv. ${ }^{b}$ & $\ln \left([\mathrm{CHO}]_{0} /[\mathrm{CHO}]_{t}\right)$ \\
\hline $\mathrm{S} 1$ & 5000 & 1 & 14 & 0.151 \\
\hline $\mathrm{S} 2$ & 5000 & 2 & 22 & 0.248 \\
\hline S3 & 5000 & 3 & 31 & 0.371 \\
\hline $\mathrm{S} 4$ & 5000 & 4 & 41 & 0.528 \\
\hline S5 & 6000 & 1 & 10 & 0.105 \\
\hline S6 & 6000 & 2 & 18 & 0.198 \\
\hline S7 & 6000 & 3 & 23 & 0.261 \\
\hline S8 & 6000 & 4 & 32 & 0.386 \\
\hline S9 & 7000 & 1 & 8 & 0.083 \\
\hline S10 & 7000 & 2 & 14 & 0.151 \\
\hline S11 & 7000 & 3 & 21 & 0.236 \\
\hline $\mathrm{S} 12$ & 7000 & 4 & 27 & 0.315 \\
\hline S13 & 8000 & 1 & 7 & 0.073 \\
\hline S14 & 8000 & 2 & 11 & 0.117 \\
\hline S15 & 8000 & 3 & 19 & 0.211 \\
\hline S16 & 8000 & 4 & 26 & 0.301 \\
\hline S17 & 9000 & 1 & 5 & 0.051 \\
\hline S18 & 9000 & 2 & 9 & 0.094 \\
\hline S19 & 9000 & 3 & 17 & 0.186 \\
\hline S20 & 9000 & 4 & 21 & 0.236 \\
\hline
\end{tabular}

Copolymerization conditions: $80{ }^{\circ} \mathrm{C}, p \mathrm{CO}_{2}{ }^{0}=20.7$ bar. ${ }^{a}$ All runs showed excellent copolymerization selectivity (>99\% carbonate linkages) based on ${ }^{1} \mathrm{H}$ NMR determination. ${ }^{b}$ Based on ${ }^{1} \mathrm{H}$ NMR analysis of the reaction mixture. 
Table S5. Kinetic Parameters for $\mathrm{CO}_{2} / \mathrm{CHO}$ Copolymerization Mediated by Dinickel

\section{Complex 6 at Various Catalyst Concentrations}

\begin{tabular}{cccccc}
\hline Entry & {$[\mathrm{CHO}]_{0}:[\mathbf{6}]_{0}$} & $\begin{array}{c}\text { Observed rate coefficient, } \\
k_{\mathrm{obs}}\left(\mathrm{h}^{-1}\right)^{a}\end{array}$ & $\begin{array}{c}\text { catalyst } \\
\ln \left(k_{\mathrm{obs}}\right)\end{array}$ & $\begin{array}{c}\text { concentrations }[6] \\
(\mathrm{M})^{b}\end{array}$ & $\ln [\mathbf{6}]$ \\
\hline $\mathrm{S} 1$ & 5000 & 0.125 & -2.077 & $2.00 \times 10^{-3}$ & -6.215 \\
$\mathrm{~S} 2$ & 6000 & 0.096 & -2.343 & $1.66 \times 10^{-3}$ & -6.401 \\
$\mathrm{~S} 3$ & 7000 & 0.085 & -2.471 & $1.43 \times 10^{-3}$ & -6.550 \\
S4 & 8000 & 0.073 & -2.621 & $1.25 \times 10^{-3}$ & -6.685 \\
S5 & 9000 & 0.067 & -2.709 & $1.11 \times 10^{-3}$ & -6.803 \\
\hline
\end{tabular}

${ }^{a}$ Calculated from the slope of the fitted regression line of Figure 3.

${ }^{b} \mathrm{~A}$ fixed amount of $\mathrm{CHO}(156.25 \mathrm{mmol})$ was used. 
Table S6. Kinetic Studies of $\mathrm{CO}_{2} / \mathrm{CHO}$ Copolymerization Mediated by Dinickel Complex 6 at Different Temperatures $\left(100,105,110,120 \text { and } 130{ }^{\circ} \mathrm{C}\right)^{a}$

\begin{tabular}{|c|c|c|c|c|}
\hline Entry & Temp $\left({ }^{\circ} \mathrm{C}\right)$ & Time/min & $\%$ CHO Conv. ${ }^{b}$ & $\ln \left([\mathrm{CHO}]_{0} /[\mathrm{CHO}]_{t}\right)$ \\
\hline $\mathrm{S} 1$ & 100 & 30 & 3 & 0.030 \\
\hline $\mathrm{S} 2$ & 100 & 60 & 13 & 0.139 \\
\hline S3 & 100 & 90 & 22 & 0.248 \\
\hline $\mathrm{S} 4$ & 100 & 120 & 27 & 0.315 \\
\hline S5 & 105 & 30 & 9 & 0.094 \\
\hline S6 & 105 & 60 & 17 & 0.186 \\
\hline S7 & 105 & 90 & 31 & 0.371 \\
\hline S8 & 105 & 120 & 37 & 0.462 \\
\hline S9 & 110 & 30 & 15 & 0.163 \\
\hline $\mathrm{S} 10$ & 110 & 60 & 30 & 0.357 \\
\hline S11 & 110 & 90 & 39 & 0.494 \\
\hline $\mathrm{S} 12$ & 110 & 120 & 47 & 0.635 \\
\hline S13 & 120 & 20 & 18 & 0.198 \\
\hline S14 & 120 & 30 & 27 & 0.315 \\
\hline $\mathrm{S} 15$ & 120 & 45 & 35 & 0.431 \\
\hline $\mathrm{S} 16$ & 120 & 60 & 43 & 0.562 \\
\hline S17 & 130 & 20 & 18 & 0.198 \\
\hline S18 & 130 & 30 & 28 & 0.329 \\
\hline S19 & 130 & 45 & 40 & 0.511 \\
\hline S20 & 130 & 60 & 51 & 0.713 \\
\hline
\end{tabular}

Copolymerization conditions: $3.125 \times 10^{-2} \mathrm{mmol}$ catalyst, $[\mathrm{CHO}]_{0} /[\mathbf{6}]_{0}=10,000, p \mathrm{CO}_{2}{ }^{0}=20.7$ bar. ${ }^{a}$ All runs showed excellent copolymerization selectivity (>99\% PCHC, $>99 \%$ carbonate linkages) based on ${ }^{1} \mathrm{H}$ NMR determination. ${ }^{b}$ Based on ${ }^{1} \mathrm{H}$ NMR analysis of the reaction mixture. 
Table S7. Kinetic Parameters for $\mathrm{CO}_{2} / \mathrm{CHO}$ Copolymerization Mediated by Dinickel Complex 6 at Various Temperatures

\begin{tabular}{cccccc}
\hline Entry & Temp $\left({ }^{\circ} \mathrm{C}\right)$ & $\begin{array}{c}\text { Observed rate } \\
\text { coefficient, } k_{\text {obs }}\left(\mathrm{h}^{-1}\right)^{a}\end{array}$ & $\ln \left(k_{\mathrm{obs}}\right)$ & $\mathrm{Temp}(\mathrm{K})$ & $\mathrm{Temp}^{-1}, 1 / \mathrm{T}\left(\mathrm{K}^{-1}\right)$ \\
\hline S1 & 100 & 0.1899 & -1.6613 & 373.15 & 0.00268 \\
S2 & 105 & 0.2576 & -1.3564 & 378.15 & 0.00264 \\
S3 & 110 & 0.3109 & -1.1683 & 383.15 & 0.00261 \\
S4 & 120 & 0.5311 & -0.6328 & 393.15 & 0.00254 \\
S5 & 130 & 0.7646 & -0.2684 & 403.15 & 0.00248 \\
\hline
\end{tabular}

${ }^{a}$ Calculated from the slope of the fitted regression line of Figure S25. 
Table S8. Crystallographic Data of Complexes 1-11

\begin{tabular}{|c|c|c|c|c|c|c|}
\hline & 1 & 2 & 3. $\mathrm{CH}_{3} \mathrm{OH}$ & $4 \cdot 2 \mathrm{C}_{3} \mathrm{H}_{6} \mathrm{O}$ & 5 & 6 \\
\hline formula & $\mathrm{C}_{37} \mathrm{H}_{42} \mathrm{~N}_{8} \mathrm{Ni}_{2} \mathrm{O}_{7}$ & $\mathrm{C}_{52} \mathrm{H}_{66} \mathrm{~F}_{6} \mathrm{~N}_{8} \mathrm{Ni}_{2} \mathrm{O}_{7}$ & $\mathrm{C}_{39} \mathrm{H}_{42} \mathrm{~F}_{6} \mathrm{~N}_{8} \mathrm{Ni}_{2} \mathrm{O}_{8}$ & $\mathrm{C}_{57} \mathrm{H}_{76} \mathrm{Cl}_{6} \mathrm{~N}_{8} \mathrm{Ni}_{2} \mathrm{O}_{9}$ & $\mathrm{C}_{37} \mathrm{H}_{36} \mathrm{Cl}_{6} \mathrm{~N}_{8} \mathrm{Ni}_{2} \mathrm{O}_{7}$ & $\mathrm{C}_{62} \mathrm{H}_{74} \mathrm{~N}_{8} \mathrm{Ni}_{2} \mathrm{O}_{7}$ \\
\hline Formula weight & 828.20 & 1146.54 & 982.22 & 1347.37 & 1034.86 & 1160.71 \\
\hline Temp (K) & $150(2)$ & $150(2)$ & $200(2)$ & $150(2)$ & $150(2)$ & $150(2)$ \\
\hline Crystal system & Monoclinic & Orthorhombic & Monoclinic & Triclinic & Triclinic & Triclinic \\
\hline Space group & $I 2 / \mathrm{a}$ & $P$ ca2 1 & $P 2(1) / \mathrm{n}$ & $P-1$ & $P-1$ & $P-1$ \\
\hline $\mathrm{a}(\AA)$ & $17.519(12)$ & $27.962(5)$ & $12.3337(19)$ & $11.4081(15)$ & $10.941(4)$ & $12.832(3)$ \\
\hline $\mathrm{b}(\AA)$ & $14.845(10)$ & $11.672(2)$ & $12.287(2)$ & $14.518(2)$ & $12.432(5)$ & $14.112(4)$ \\
\hline $\mathrm{c}(\AA)$ & $29.264(19)$ & $16.506(3)$ & $28.047(5)$ & $20.123(3)$ & $17.318(9)$ & $18.461(5)$ \\
\hline$\alpha(\mathrm{deg})$ & 90 & 90 & 90 & $92.316(6)$ & $87.378(17)$ & $81.809(12)$ \\
\hline$\beta(\mathrm{deg})$ & $94.10(4)$ & 90 & $99.834(5)$ & $101.095(5)$ & $75.392(19)$ & $70.255(10)$ \\
\hline$\gamma(\mathrm{deg})$ & 90 & 90 & 90 & $90.655(5)$ & $85.68(4)$ & $73.872(12)$ \\
\hline$V\left(\AA^{3}\right)$ & 7591(9) & $5387.1(17)$ & $4187.9(12)$ & $3267.2(8)$ & $2272.1(17)$ & $3018.3(14)$ \\
\hline Z & 8 & 4 & 4 & 2 & 2 & 2 \\
\hline$D_{\text {calc }}\left(\mathrm{Mg} / \mathrm{m}^{3}\right)$ & 1.449 & 1.414 & 1.558 & 1.370 & 1.513 & 1.277 \\
\hline$\mu(\operatorname{Mo~K} \alpha)\left(\mathrm{mm}^{-1}\right)$ & 1.051 & 0.778 & 0.988 & 0.879 & 1.236 & 0.681 \\
\hline$F(000)$ & 3456 & 2400 & 2024 & 1408 & 1056 & 1228 \\
\hline Reflections collected & 103582 & 81817 & 52636 & 49924 & 48647 & 45372 \\
\hline No. of parameters & 494 & 701 & 578 & 749 & 551 & 716 \\
\hline Indep. reflns $\left(R_{\text {int }}\right)$ & $6681(0.0768)$ & $9373(0.1732)$ & $7438(0.0943)$ & $10658(0.0990)$ & $7403(0.0754)$ & $10069(0.1023)$ \\
\hline$R 1[I>2 \sigma(I)]$ & 0.0363 & 0.0600 & 0.0539 & 0.0803 & 0.0583 & 0.1294 \\
\hline $\mathrm{w} R 2[I>2 \sigma(I)]$ & 0.0824 & 0.0888 & 0.1339 & 0.2284 & 0.1353 & 0.2930 \\
\hline Goodness-of-fit on $F^{2}$ & 1.087 & 1.024 & 0.843 & 1.009 & 0.892 & 1.161 \\
\hline
\end{tabular}


Table S8. Crystallographic Data of Complexes 1-11 (Cont'd)

\begin{tabular}{|c|c|c|c|c|c|}
\hline & 7 & 8 & $9 \cdot \mathrm{C}_{4} \mathrm{H}_{8} \mathrm{O}$ & 10 & $11 \cdot 0.5 \mathrm{CH}_{3} \mathrm{OH}$ \\
\hline formula & $\mathrm{C}_{47} \mathrm{H}_{46} \mathrm{~N}_{8} \mathrm{Ni}_{2} \mathrm{O}_{7}$ & $\mathrm{C}_{63} \mathrm{H}_{78} \mathrm{~N}_{8} \mathrm{Ni}_{2} \mathrm{O}_{9}$ & $\mathrm{C}_{54} \mathrm{H}_{60} \mathrm{~N}_{8} \mathrm{Ni}_{2} \mathrm{O}_{10}$ & $\mathrm{C}_{63} \mathrm{H}_{72} \mathrm{~F}_{6} \mathrm{~N}_{8} \mathrm{Ni}_{2} \mathrm{O}_{7}$ & $\mathrm{C}_{62.50} \mathrm{H}_{68} \mathrm{~F}_{10} \mathrm{~N}_{8} \mathrm{Ni}_{2} \mathrm{O}_{7.50}$ \\
\hline Formula weight & 952.34 & 1208.75 & 1098.52 & 1284.70 & 1358.66 \\
\hline Temp (K) & $150(2)$ & $150(2)$ & 200(2) K & $150(2)$ & $150(2)$ \\
\hline Crystal system & Monoclinic & Trigonal & Monoclinic & Monoclinic & Triclinic \\
\hline Space group & $P 2(1) / \mathrm{c}$ & $P 3 \mathrm{c} 1$ & $P 2(1) / \mathrm{n}$ & $P 2(1) / \mathrm{c}$ & $P-1$ \\
\hline $\mathrm{a}(\AA)$ & $13.2828(10)$ & $20.331(5)$ & $13.0681(10)$ & $15.805(3)$ & $16.2663(19)$ \\
\hline $\mathrm{b}(\AA)$ & $22.915(2)$ & $20.331(5)$ & $14.3059(9)$ & $35.736(7)$ & $19.514(2)$ \\
\hline c $(\AA)$ & $14.6547(14)$ & $27.922(5)$ & $28.053(2)$ & $13.079(3)$ & $21.946(2)$ \\
\hline$\alpha(\operatorname{deg})$ & 90 & 90 & 90 & 90 & 104.974(4) \\
\hline$\beta(\operatorname{deg})$ & $105.342(3)$ & 90 & $102.469(2)$ & $92.753(7)$ & $93.501(4)$ \\
\hline$\gamma(\operatorname{deg})$ & 90 & 120 & 90 & 90 & $110.021(4)$ \\
\hline$V\left(\AA^{3}\right)$ & $4301.6(6)$ & $9995(5)$ & $5120.8(6)$ & $7379(3)$ & $6236.5(11)$ \\
\hline $\mathrm{Z}$ & 4 & 6 & 4 & 4 & 4 \\
\hline$D_{\text {calc }}\left(\mathrm{Mg} / \mathrm{m}^{3}\right)$ & 1.471 & 1.205 & 1.425 & 1.156 & 1.447 \\
\hline$\mu(\mathrm{Mo} \mathrm{K} \alpha)\left(\mathrm{mm}^{-1}\right)$ & 0.939 & 0.622 & 0.803 & 0.575 & 0.694 \\
\hline$F(000)$ & 1984 & 3840 & 2304 & 2688 & 2820 \\
\hline Reflections collected & 87473 & 187376 & 69389 & 97208 & 117032 \\
\hline No. of parameters & 581 & 755 & 652 & 788 & 1645 \\
\hline Indep. reflns $\left(R_{\text {int }}\right)$ & $7970(0.0615)$ & $11709(0.1036)$ & $9017(0.0380)$ & $12859(0.1267)$ & 23982(0.0842) \\
\hline$R 1[I>2 \sigma(I)]$ & 0.0417 & 0.0949 & 0.0460 & 0.1211 & 0.0466 \\
\hline $\mathrm{w} R 2[I>2 \sigma(I)]$ & 0.0867 & 0.2369 & 0.1404 & 0.3043 & 0.1111 \\
\hline Goodness-of-fit on $F^{2}$ & 1.078 & 1.062 & 0.816 & 2.274 & 1.012 \\
\hline
\end{tabular}

